\title{
Temporal modulation of spike-timing-dependent plasticity
}

\author{
Robert C. Froemke ${ }^{1 *}$, Dominique Debanne ${ }^{2,3}$ and Guo-Qiang $B i^{4,5}$ \\ Molecular Neurobiology Program, Departments of Otolaryngology and Physiology/Neuroscience, The Helen and Martin Kimmel Center for Biology and Medicine, \\ Skirball Institute of Biomolecular Medicine, New York University School of Medicine, New York, NY, USA \\ 2 Institut National de la Santé et de la Recherche Médicale U641, Marseille, France \\ 3 Faculté de Médecine Nord, Université de la Méditerranée, Marseille, France \\ ${ }^{4}$ Department of Neurobiology and Center for Neuroscience and Center for the Neural Basis of Cognition, University of Pittsburgh School of Medicine, Pittsburgh, \\ PA, USA \\ ${ }^{5}$ Hefei National Laboratory for Physical Sciences at the Microscale and School of Life Sciences, University of Science and Technology of China, Hefei, Anhui, China
}

\section{Edited by:}

Per Jesper Sjöström, University

College London, UK

\section{Reviewed by:}

Samuel S. H. Wang, Princeton

University, USA

Kevin Bender, Oregon Health and

Science University, USA

Per Jesper Sjöström, University

College London, UK

*Correspondence:

Robert C. Froemke, Molecular Neurobiology Program, Departments of Otolaryngology and Physiology/

Neuroscience, The Helen and Martin

Kimmel Center for Biology and

Medicine, Skirball Institute of

Biomolecular Medicine, New York

University School of Medicine,

New York, NY 10016, USA.

e-mail: robert.froemke@med.nyu.edu
Spike-timing-dependent plasticity (STDP) has attracted considerable experimental and theoretical attention over the last decade. In the most basic formulation, STDP provides a fundamental unit -a spike pair - for quantifying the induction of long-term changes in synaptic strength. However, many factors, both pre- and postsynaptic, can affect synaptic transmission and integration, especially when multiple spikes are considered. Here we review the experimental evidence for multiple types of nonlinear temporal interactions in STDP, focusing on the contributions of individual spike pairs, overall spike rate, and precise spike timing for modification of cortical and hippocampal excitatory synapses. We discuss the underlying processes that determine the specific learning rules at different synapses, such as postsynaptic excitability and short-term depression. Finally, we describe the success of efforts toward building predictive, quantitative models of how complex and natural spike trains induce long-term synaptic modifications.

Keywords: cortex, hippocampus, LTD, LTP, model, spikes, STDP, synaptic plasticity

\section{INTRODUCTION}

Synaptic plasticity is essential for the organization and function of neural circuits. Long-term changes in synaptic strength have been described for many systems, ranging from the invertebrate neuromuscular junction to the mammalian hippocampus and neocortex (Malenka and Nicoll, 1999; Zucker, 1999). Correspondingly, it is believed that there are several important consequences of longterm synaptic modification depending on when and where synaptic modifications occur, including neural development (Katz and Shatz, 1996), cortical map formation, and reorganization (Cruikshank and Weinberger, 1996; Buonomano and Merzenich, 1998; Kilgard et al., 2002), alteration of receptive field properties (Fregnac and Shulz, 1999; Froemke et al., 2007), perceptual learning (Gilbert, 1998), behavioral conditioning (Schafe et al., 2001), and memory encoding and storage (Martin et al., 2000). It is therefore critical to understand the general rules by which synapses are changed in response to various patterns of neural activity.

Most types of long-term synaptic modification can be formulated in terms of Hebbian learning. The neurophysiological postulate of Hebb (1949) has exerted tremendous influence on the study of synaptic plasticity. Hebb's idea was that associative learning such as Pavlovian conditioning (Pavlov, 1927) could be represented in a neural circuit by changes in the patterns of synaptic connections, a concept that was inspired by the experimental findings of Lorente de Nó (1938). In particular, Hebb believed that persistent activation of a postsynaptic neuron by a presynaptic input should lead to a strengthening of the synapse between the two cells. This hypothesis for associative synaptic plasticity was later extended by Stent (1973), who suggested the converse idea for bidirectional synaptic modification - that persistent failure of a presynaptic input to activate the postsynaptic neuron should lead to weakening of that synapse.

In the years that followed, Hebb's postulate exerted a profound influence on theoretical and experimental neurophysiology. Spurred by the discovery of hippocampal long-term potentiation (LTP) by Bliss and Lømo (1973), along with other experimental reports of changes in cortical activity (Bindman et al., 1962), many different theoretical frameworks for long-term synaptic modification were proposed, such as the covariance model (Stanton and Sejnowski, 1989) or temporal difference learning (Sutton and Barto, 1998; Rao and Sejnowski, 2001).

One of the most successful paradigms for the study of long-term synaptic plasticity is the Bienenstock-Cooper-Munro (BCM) sliding threshold model (Bienenstock et al., 1982). In this scheme, the sign and degree of synaptic modification is a nonlinear function of postsynaptic spike rate. When the postsynaptic spike frequency is above a certain threshold $\left(\theta_{\mathrm{m}}\right)$ LTP is induced, while long-term depression (LTD) is induced when the firing rate is below $\theta_{\mathrm{m}}$ but greater than zero. The value of $\theta_{\mathrm{m}}$ is not fixed, but varies so as to prevent runaway potentiation or depression to saturation. The BCM model was originally proposed as a method by which synaptic modifications could result in the development and plasticity 
of ocular dominance and orientation tuning in cat and monkey visual cortex. Since its description, an abundance of experimental evidence for the BCM model has been obtained in many preparations, including the hippocampus and visual cortex (Kirkwood et al., 1993, 1995). Additionally, other properties of the postsynaptic cell beyond firing rate lead to biphasic functions reminiscent of the characteristic BCM curve, including presynaptic input rate, postsynaptic depolarization, inhibitory tonus, and internal calcium concentration ( $\left[\mathrm{Ca}^{2+}\right]_{\mathrm{i}}$ ) (Artola et al., 1990; Hansel et al., 1997; Yang et al., 1999). The BCM model is Hebbian in that the requisite postsynaptic spiking presumably results from activation of a subset of presynaptic inputs. However, because the BCM model is a rate-based learning rule, it does not necessarily require the precise temporal ordering of pre- and postsynaptic activity. In particular, the BCM model predicts that for modest firing rates, LTD is induced even if the presynaptic cell routinely takes part in firing the postsynaptic cell.

Recently, Hebbian learning at the synaptic level has been recast in terms of correlated pre- and postsynaptic spiking, a formulation more consistent with Hebb's original thesis. Over the last few decades, some studies had found that the temporal order of preand postsynaptic activity was a crucial parameter for induction of both LTP and LTD (Baranyi and Fehér, 1981; Levy and Steward, 1983; Kelso et al., 1986; Gustafsson et al., 1987; Zador et al., 1990; Abbott and Blum, 1996; Sourdet and Debanne, 1999). Then in the 1990s, a number of groundbreaking papers showed that in a variety of preparations, repetitive stimulation with pairs of pre- and postsynaptic action potentials (pre/post pairs) led to induction of long-term synaptic plasticity. Importantly, the precise pre/post spike timing controlled both the sign and magnitude of synaptic modification (Debanne et al., 1994; Bell et al., 1997; Markram et al., 1997; Bi and Poo, 1998; Debanne et al., 1998; Zhang et al., 1998), and therefore this phenomenon was dubbed "spike-timing-dependent plasticity” (STDP) (Abbott and Nelson, 2000; Song et al., 2000).

Spike-timing-dependent plasticity has several properties that make it a useful protocol for investigating long-term synaptic plasticity. First, STDP is quantifiable. This allows for accurate predictions of synaptic plasticity spike for spike, enabling experiments to be designed that carefully measure the sign and degree of synaptic modification induced by complex patterns of neural activity (Figure 1). For this reason, STDP has rapidly become a popular choice for theoretical studies of synaptic plasticity in neural networks (Gerstner et al., 1996; Sejnowski, 1999; Senn et al., 1999; Abbott and Nelson, 2000; Gutig and Sompolinsky, 2006; Pfister and Gerstner, 2006; Morrison et al., 2008; Urakubo et al., 2008). Second, STDP is a robust phenomenon. The shape and size of the time window for induction of both LTP and LTD of excitatory synapses is remarkably conserved across different preparations (Abbott and Nelson, 2000; Dan and Poo, 2006), with a few notable exceptions (Bell et al., 1997; Egger et al., 1999; Letzkus et al., 2006; Sjöström and Häusser, 2006). Finally, STDP provides an intuitive cellular mechanism for associative learning and behavioral conditioning. The correlation between spike timing and the sign/magnitude of response modification is strikingly similar to that observed in classic conditioning experiments (Pavlov, 1927), albeit on a different time scale. STDP may also represent a basic neurophysiological correlate of the principle of causality (Berninger and $\mathrm{Bi}, 2002$ ), responsible for a priori temporal organization of mental events, in the sense of Kant (1781). For these reasons, in the last 15 years there has been an explosion in the number of both experimental and theoretical studies of STDP, and in turn the unusually close collaboration between experiment and theory has been an important reason for the success of the STDP subfield.

In this review, we summarize the results of experiments on the timing requirements for STDP, focusing mainly on excitatory neocortical and hippocampal synapses in brain slices and culture. We detail the effects of temporal modulation during STDP induction, including variations in number of pre/post pairings, spike frequency, and precise spike timing. While other parameters such as dendritic location of synaptic inputs or local neuromodulatory status are also important, these variables are more fully described in other reviews in this issue (Froemke et al., 2007; Seol et al., 2007). The overall goal of these parametric experiments is a complete, predictive model of how complex patterns of pre- and postsynaptic activity modify synaptic strength. Such models will be required to support large-scale efforts to simulate brain circuitry (Markram, 2006; Izhikevich and Edelman, 2007), and to inform treatments for impairments of learning and memory in nervous system disorders (Merzenich et al., 1996; Tallal et al., 1996).

\section{FIRST-ORDER STDP INDUCED WITH SPIKE PAIRS}

Computationally, STDP is ideal as a synaptic learning rule, as it provides a basic discrete unit for long-term modification: the spike pair. Repetitive presentation of single pre- and postsynaptic spike pairs induces spike-timing-dependent LTP and LTD in hippocampus (Debanne et al., 1998; Bi and Poo, 1998; Nishiyama et al., 2000; Lin et al., 2003; Tsukada et al., 2005; Figures 1 and 2A), neocortex (Feldman, 2000; Sjöström et al., 2001; Froemke and Dan, 2002; Birtoli and Ulrich, 2004; Zilberter et al., 2009; Figure 2B), and other systems (Bell et al., 1997; Zhang et al., 1998; Pawlak and Kerr, 2008). In these studies, usually 50-100 pairs of spikes are evoked at low frequency $(0.1-5 \mathrm{~Hz})$ by focal extracellular stimulation and/ or direct depolarizing current injection, where a critical parameter for determining synaptic modification is the time interval between the pre- and postsynaptic spikes.

At most glutamatergic synapses, LTP is induced when the presynaptic neuron fires before the postsynaptic neuron (pre $\rightarrow$ post pairing at positive time intervals), and LTD is induced if the postsynaptic cell fires before the presynaptic cell (post $\rightarrow$ pre pairing at negative time intervals), such that the degree of synaptic modification depends on the relative time between the two spikes or sets of spikes. Although there are many types of STDP learning rules (Abbott and Nelson 2000; Caporale and Dan 2008), the hallmark of excitatory STDP is a time window of approximately 0-20 ms for the induction of LTP and -1 to $-100 \mathrm{~ms}$ for LTD, outside of which no synaptic modification occurs (Figures 1 and 2). Uncorrelated pre/post spiking at low firing rates generally leads to LTD because the integrated area under the spike timing window for depression is usually larger than that for potentiation. It is still unclear what cellular factors determine these timing requirements, especially the sharp transition point that occurs around time zero. There is evidence that, generally, the window for LTP is set by the activation kinetics of NMDA receptors (Kampa et al., 2004; Urakubo et al., 2008), but the LTD window seems to be more variable 

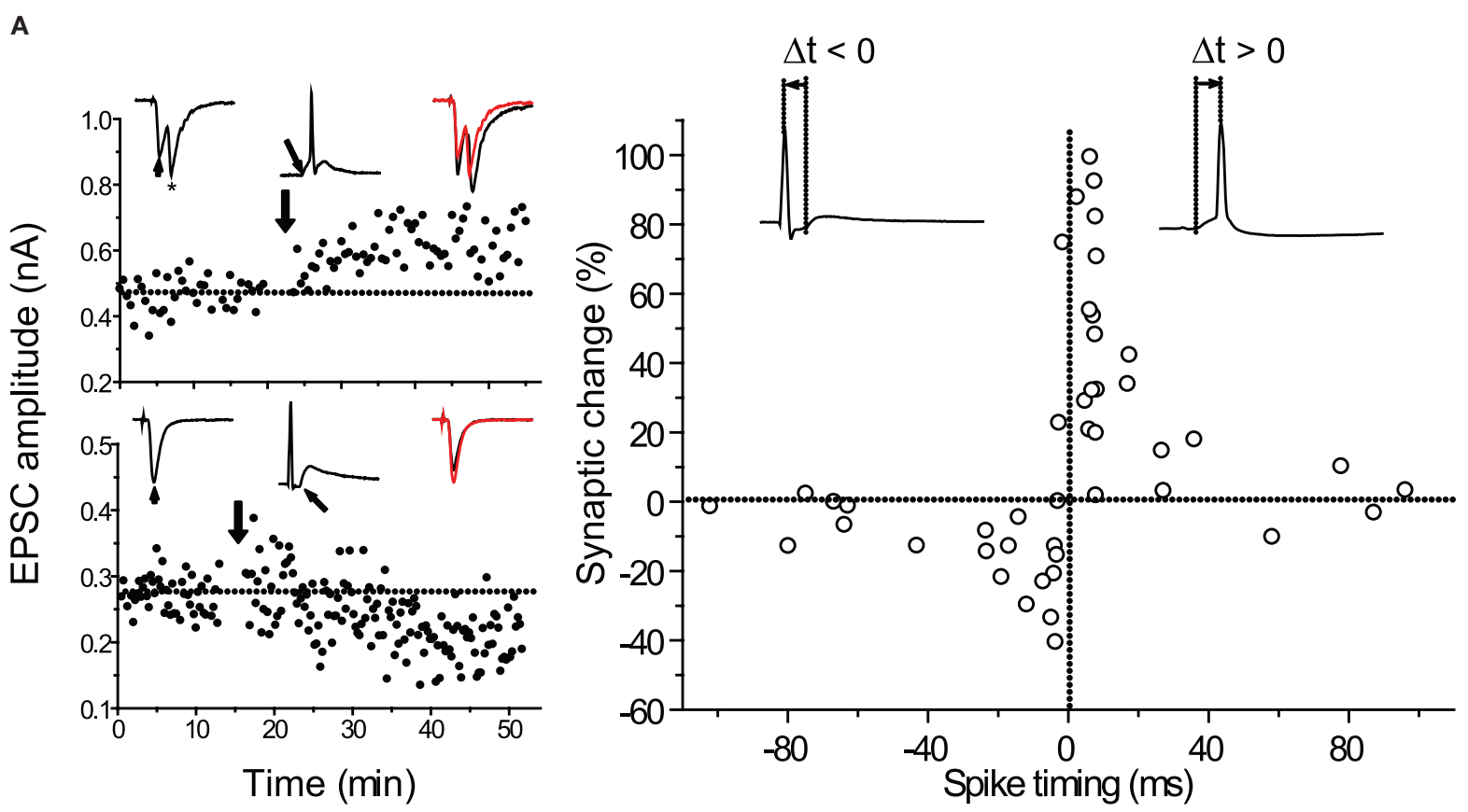

B
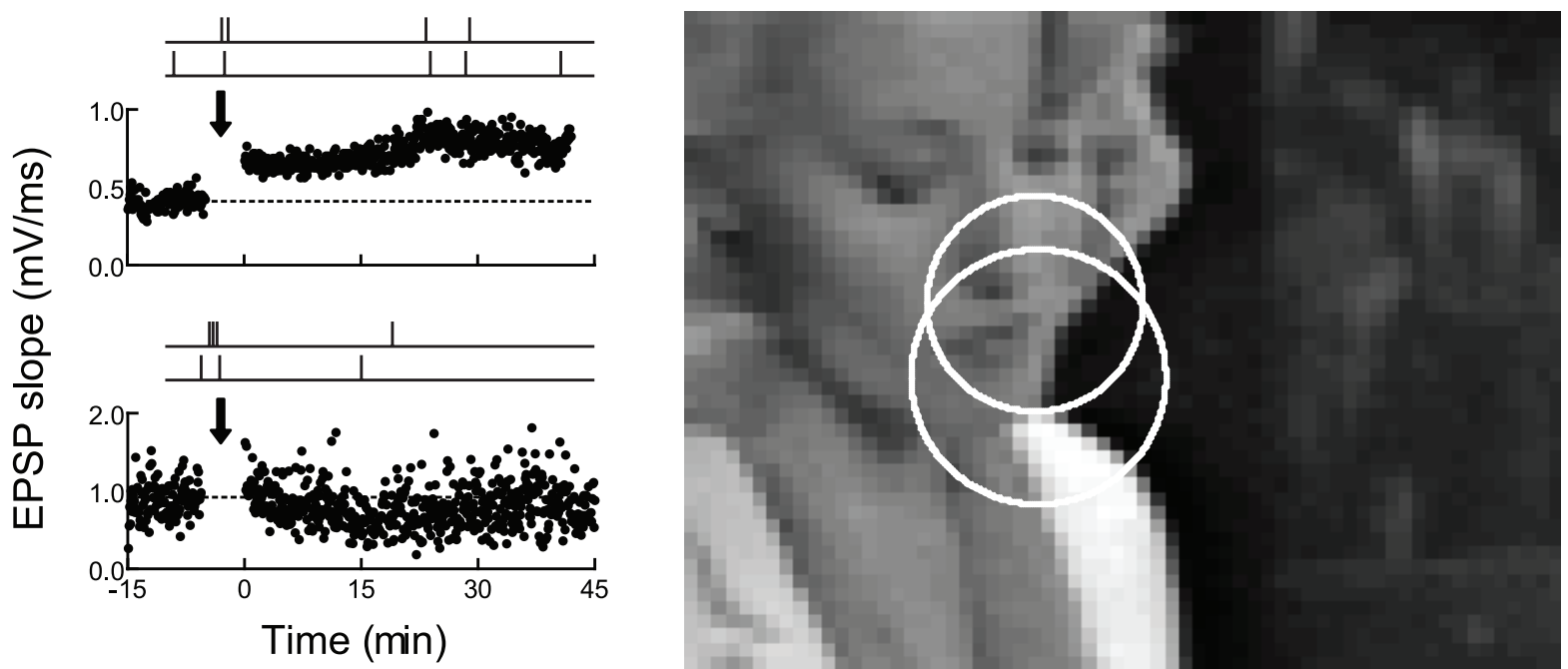

FIGURE 1 | Spike-timing-dependent plasticity can be used to predict how complex spike trains induce long-term changes in synaptic strength. (A) Timing requirements for STDP induction in excitatory neurons from low-density hippocampal cultures. Left, examples of LTP induced by pre $\rightarrow$ post pairing (top) and LTD induced by post $\rightarrow$ pre pairing (bottom) at short time intervals. Right, critical time window for synaptic modifications. Each circle represents one experiment. Curves, single exponential fits to the data. From Bi and Poo (1998, 2001). (B) How does the STDP learning rule for spike pairs need to be modified for predicting the effects of complex spike trains? Left, examples of LTP (top) and LTD (bottom) induced by natural spike train fragments in slices of young rat visual cortex. Right, scene from a movie used to obtain natural spike trains from the cat visual cortex in vivo. From Froemke and Dan (2002). across different synapses, and may require such diverse processes as $\mathrm{Ca}^{2+}$-dependent postsynaptic NMDA receptor suppression, presynaptic NMDA autoreceptor activation, endocannabinoid release, and metabotropic glutamate receptors, depending on cell type, strength of postsynaptic depolarization, pre/post spike rates, and location of synaptic input (Senn et al., 1999; Abarbanel et al., 2002; Franks and Sejnowski, 2002; Karmarkar and Buonomano, 2002; Shouval et al., 2002; Sjöström et al., 2003; Birtoli and Ulrich, 2004; Froemke et al., 2005; Bender et al., 2006;
Corlew et al., 2007; Urakubo et al., 2008; Feldman, 2009). While a central mechanism for STDP is $\mathrm{Ca}^{2+}$ influx through voltage-gated $\mathrm{Ca}^{2+}$ channels and NMDA receptors (Koester and Sakmann, 1998; Johnston et al., 2003; Froemke et al., 2006; Nevian and Sakmann, 2006), other processes, including dendritic excitability (Letzkus et al., 2006; Sjöström and Häusser, 2006) and $\mathrm{Ca}^{2+}$ release from internal stores (Wang et al., 2000; Larkum et al., 2003), are also implicated in STDP induction at different synapses (Nishiyama et al., 2000; Froemke et al., 2010). It should be highlighted that 

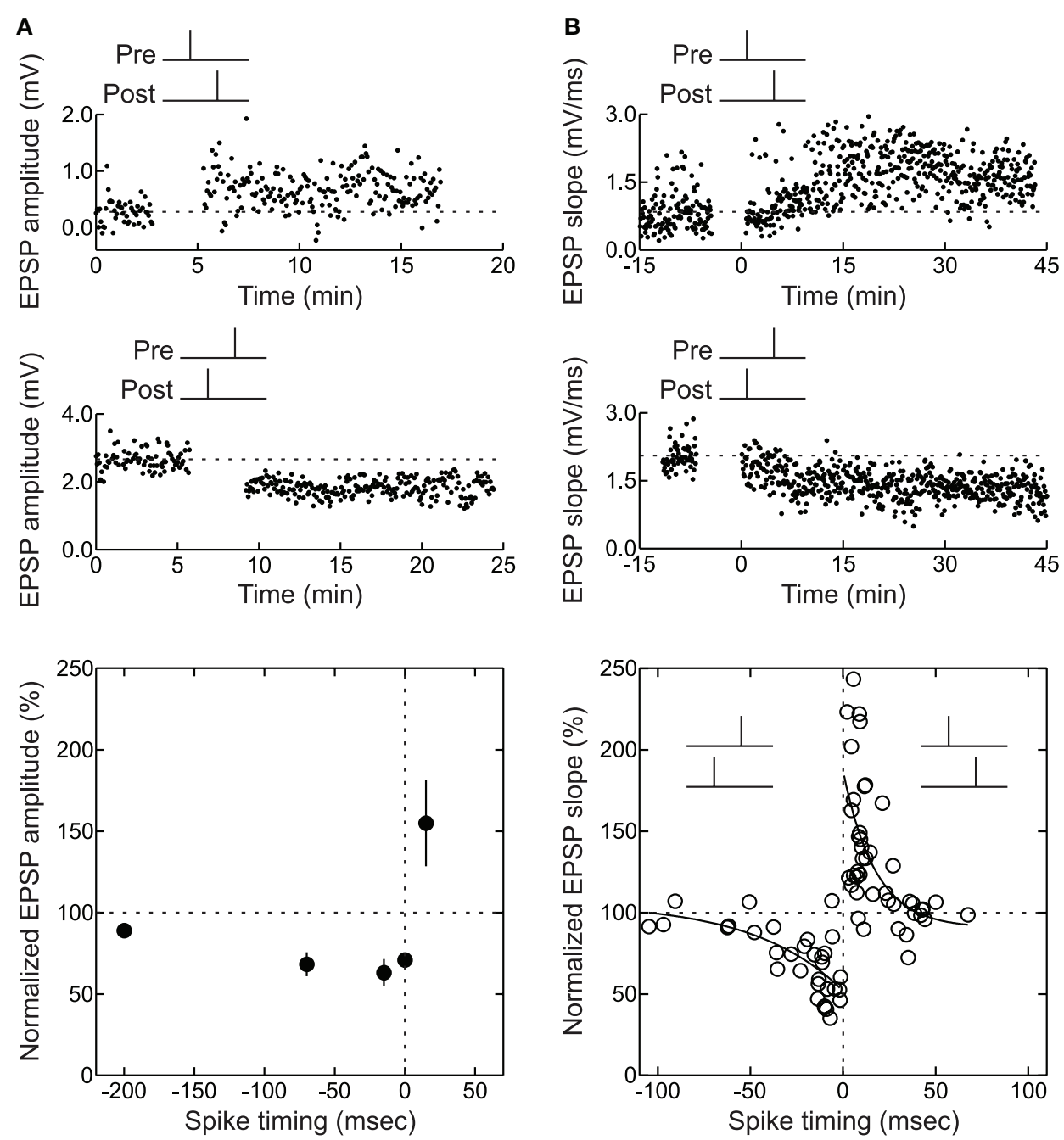

FIGURE 2 | Pre/post pairing induces STDP at hippocampal and neocortical synapses. (A) STDP induced at CA3-CA3 synapses in hippocampal slice cultures. Top, LTP induced by pre $\rightarrow$ post pairing ( $\Delta t=15 \mathrm{~ms}$ ). Center, LTD induced by post $\rightarrow$ pre spiking $(\Delta t=-70 \mathrm{~ms})$. Bottom, time window

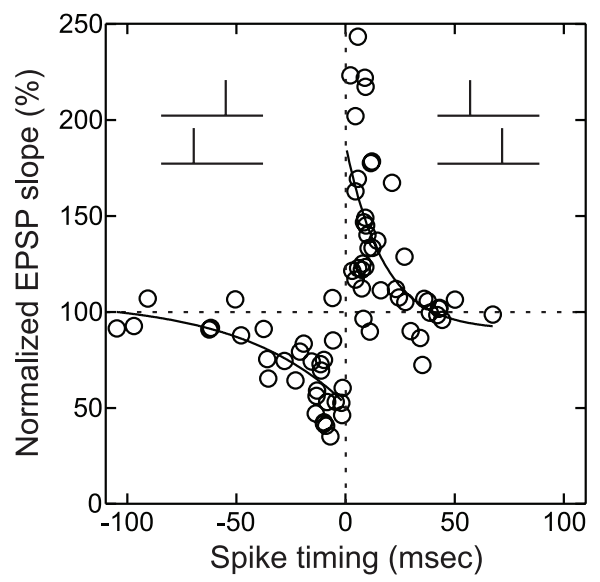

for pre/post pairing to induce synaptic modifications. From Debanne et al. (1998). (B) As in A, but for layer 2/3 connections in acute brain slices of young rat visual cortex. From Froemke and Dan (2002) and Froemke et al. (2006).

STDP induction seems to be somewhat sensitive to technical details, in terms of spike number, spike timing, spike frequency, and underlying mechanisms such as inhibitory regulation. This seems especially true for CA1 pyramidal cells in hippocampal slices, where it remains controversial what is minimally required for LTP and LTD (Pike et al., 1999; Nishiyama et al. 2000; Meredith et al., 2003; Wittenberg and Wang, 2006; Buchanan and Mellor, 2007; but see Campanac and Debanne, 2008).

This millisecond-scale time window for pre/post pairings to induce LTP or LTD forms the basis of the STDP learning rule for a given synaptic connection. To quantify the effect of pre/post pairing more precisely, both the pre $\rightarrow$ post and post $\rightarrow$ pre data can be fitted with single exponential functions: $\Delta w=A e^{-|\Delta t| / \tau}$, where $\Delta w$ is the percentage change in synaptic weight, $\Delta t$ is the pre/post spike interval, and $A$ and $\tau$ are two free parameters found by fitting the data, representing the scaling factor and time constant of the exponential function, respectively (Song et al., 2000; Bi and Poo, 2001; Froemke and Dan, 2002; Figures 1 and 2). Biologically, STDP is unlikely to be a true single exponential process, but these exponential fits are a convenient way to adequately formalize STDP using a low parameter model.

Here we refer to this formulation of STDP, in which only the intervals between each pre/post pairing are considered for determination of net synaptic modification, as the "history-independent" model. While sometimes found to be satisfactory when overall spike rates are relatively low $(<10 \mathrm{~Hz})$, in general the history-independent model provides poor estimates of the effects of more complex spike trains, even when one additional spike is added to a pre/post pair, i.e., using spike triplets instead of spike pairs to induce STDP (Pfister and Gerstner, 2006). Comparisons between the predicted and actual effects of spike trains on synaptic strength show that the predictions of the independent model are generally poor and 
sometimes non-physiological (Sjöström et al., 2001; Froemke and Dan, 2002; Froemke et al., 2006; Butts et al., 2007). To better predict how arbitrary spike trains change synaptic strength in terms of spike pair contributions, there are three initial questions: (1) whether STDP requires the full number of 60-100 pre/post pairings repeated over several minutes for successful induction of LTP or LTD; (2) to what degree LTP and LTD saturate; and (3) how individual pairwise effects are combined or integrated to determine the net change in synaptic strength.

First we will consider how many pairing events are needed for STDP. The amount of LTP or LTD induced by repetitive spike pairing is usually measured $10+$ minutes post-induction. However, for some cells, significant changes in synaptic strength can be observed to occur immediately after termination of spike pairing, while for other cells, synaptic modifications were delayed for several minutes. Across synapses, it seems that approximately one-third of synapses are changed immediately after pairing, one-third are changed with a delay of several minutes, and one-third show both immediate and delayed changes in synaptic strength (Magee and Johnston, 1997; Markram et al., 1997; Debanne et al., 1999; Sjöström et al., 2001; Hoffman et al., 2002; Froemke et al., 2006).
For those experiments in which LTP or LTD was rapidly induced, trial-by-trial examination of EPSPs during the spike pairing procedure might indicate how many pairings are needed to increase or decrease synaptic efficacy. For STDP of layer 2/3 lateral connections in slices of the young rat visual cortex, in which 60 pre/post pairs were presented at $0.2 \mathrm{~Hz}$ for $5 \mathrm{~min}$ (Froemke et al., 2006), there was a progressive increase in synaptic strength with repetitive pre $\rightarrow$ post spike pairing. About half of those pre $\rightarrow$ post experiments that could be analyzed showed a steady increase in EPSP size with continued spike pairing (Figure 3A), while other cells showed a pronounced, significant stepwise increase in synaptic strength at some point during the $5 \mathrm{~min}$ of spike pairing (Figure 3B). Remarkably, it appeared that as little as 1 min of conditioning (12 pairs) was sufficient to induce a modest but significant amount of potentiation (Figure 3D). The development of LTD induced with post $\rightarrow$ pre spike pairs, on the other hand, required more pairings than LTP. In general, while one minute of pre $\rightarrow$ post spike pairing induced significant LTP, LTD required 4 min of post $\rightarrow$ pre pairing to develop (Figure 3C and D). In general, induction of LTD requires more prolonged periods of activity than LTP (Dudek and Bear, 1992; Yang et al., 1999; Froemke et al., 2006; Wittenberg and Wang, 2006).
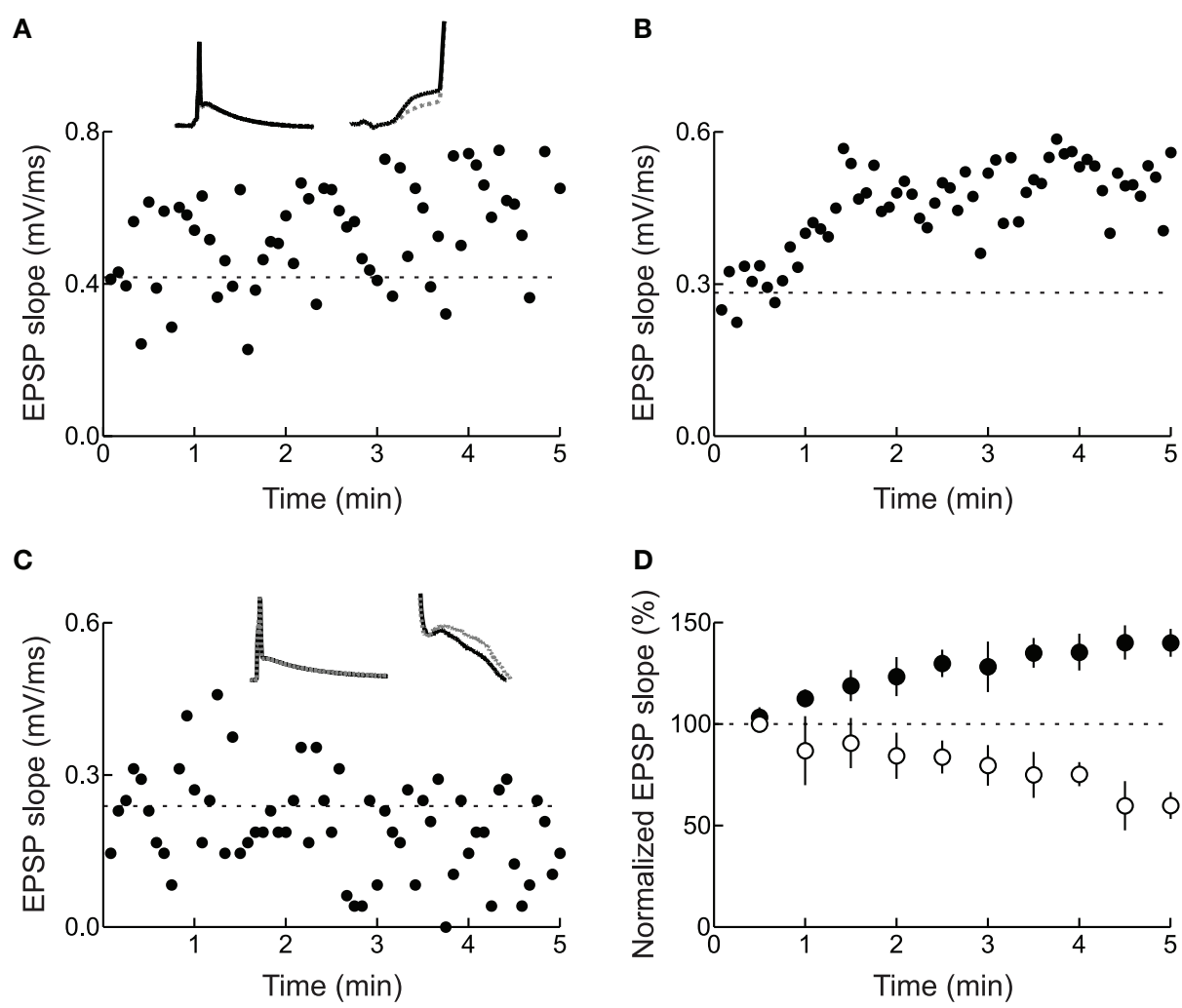

FIGURE 3 | Changes in synaptic strength during pre/post spike pairing. (A) Example of continual increase in initial slope during pre $\rightarrow$ post spike pairing with 60 pairs. This cell did not display a significant $(p<0.05)$ increase in slope from minute to minute. Dashed line, average initial slope during baseline period before conditioning. Left inset, average postsynaptic response during first six (gray dashed line) and last six (solid black line) pairings. Right inset, blow-up showing the increase in EPSP slope. (B) Example of a stepwise increase in initial slope induced by pre $\rightarrow$ post spike pairing. This cell showed a significant

increase in initial slope from first to second minute of pairing. (C) Example of decrease in initial slope during post $\rightarrow$ pre spike pairing with 60 pairs. Dashed line, average initial slope of first six post $\rightarrow$ pre pairings. Left inset, average postsynaptic response during first six (gray dashed line) and last six (solid black line) pairings. Right inset, blow-up showing the decrease in EPSP slope. (D) Summary of conditioning period for cells that displayed significant changes in synaptic strength immediately after pre/post pairing. From Froemke et al. (2006). 
The observation that some synapses were potentiated or depressed during or immediately after conditioning suggests that fewer than 60 pre/post spike pairs can induce long-term changes in synaptic strength. In the optic tectum of the tadpole in vivo, spiketiming-dependent LTP was maximal after 80-200 pairs, but 20 pre $\rightarrow$ post spike pairs induced a moderate amount of potentiation on average (Zhang et al., 1998). In cortex, a small number of pre/ post pairs could induce either LTP or LTD, but LTP required fewer spike pairs $(<15)$ than LTD (Froemke et al., 2006). Surprisingly, for those cells that expressed significant LTP, the magnitude of potentiation was independent of the number of pre/post pairings (Figure 4). One interpretation of these data is that the amount of LTP depends on the pre/post spike interval, while the probability of LTP induction depends on the number of spike pair repetitions. Such a scenario could imply certain co-operativity of LTP among multiple synaptic sites receiving the same inputs (Harvey and Svoboda, 2007). The magnitude of LTD, on the other hand, increased gradually with more post $\rightarrow$ pre pairs.
Alternatively, at individual pre- and postsynaptic loci, expression of LTP or LTD might be all-or-none, possibly down to the level of single glutamate receptors (Peterson et al., 1998; Lee et al., 2000; O'Connor et al., 2005; but see Tanaka et al., 2008 and Enoki et al., 2009). In this case, the magnitude of synaptic modification would be fixed at a given synapse, suggesting that both pre/post spike timing interval and the total number of pairings jointly determine the probability of induction. The time course of synaptic modification might still appear graded if enough separate inputs contribute to the overall synaptic response, with induction of synaptic plasticity staggered over multiple sites.

A third, non-mutually exclusive possibility is that synaptic strength is initially modified with a smaller number of spike pairs, but the duration of these changes depends on the total number of pairings. A larger number of spike pairings would then consolidate synaptic modifications and extend their duration. Without the occurrence of these additional events within some interval, synaptic modifications would not persist. For example, interleaving pre/post
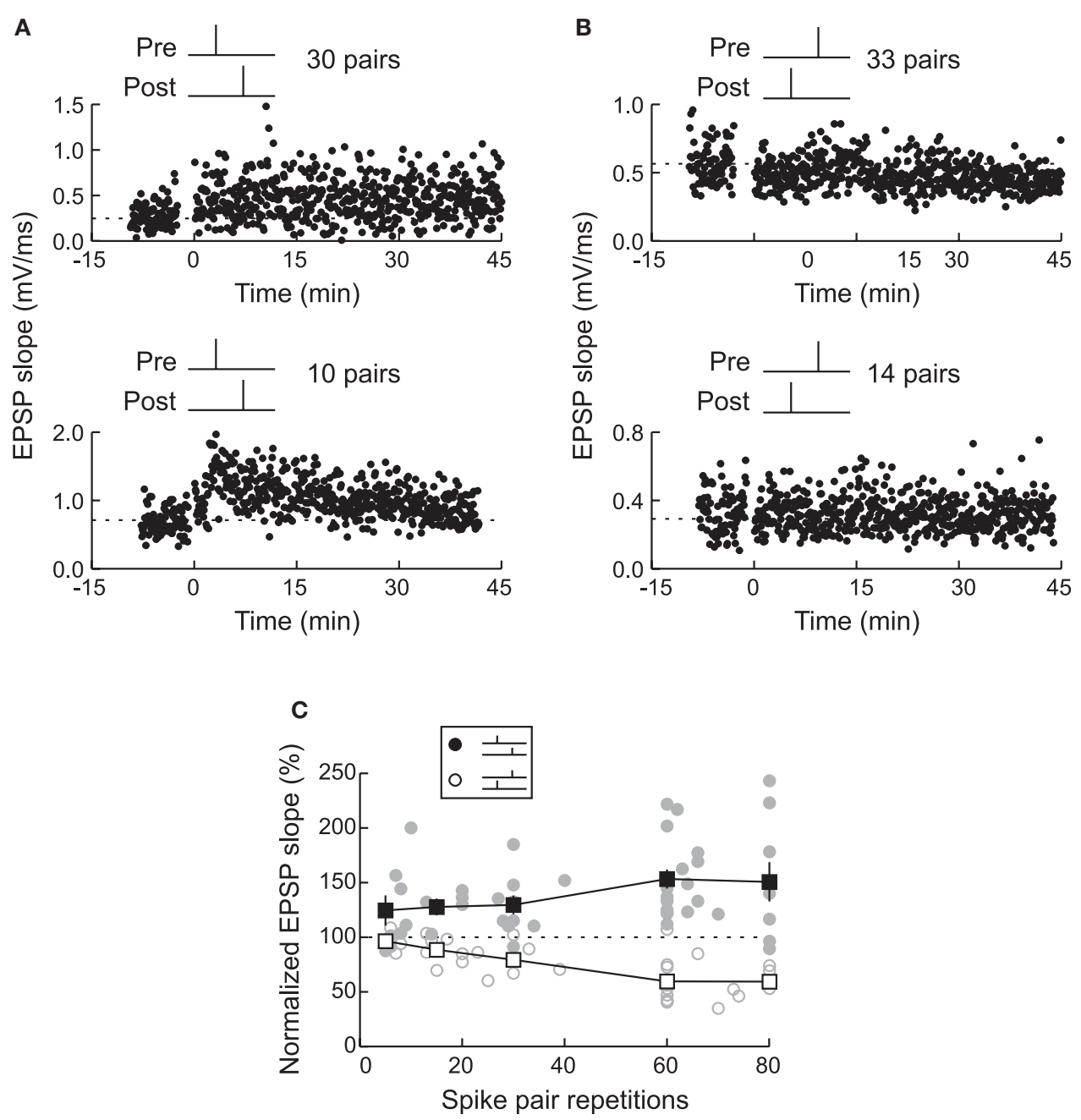

FIGURE 4 |Timing-dependent LTP requires fewer spike pairs than timing-dependent LTD. (A) LTP was induced by 30 pre $\rightarrow$ post spike pairs (top) or 10 pre $\rightarrow$ post pairs (bottom). (B) LTD was induced by 33 post $\rightarrow$ pre spike pairs (top), but not by 14 post $\rightarrow$ pre pairs (bottom). (C) The magnitude of LTP and LTD depended on the number of pre/post spike pairs. LTP required a smaller number of pairings (filled symbols) than LTD (open symbols). Each circle represents one experiment and squares represent summary data. From Froemke et al. (2006). 
pairings with unpaired pre- or postsynaptic activation prevents induction of LTP (Bauer et al., 2001). In Xenopus optic tectum, synaptic modifications induced by spike pairing can be washed out or extinguished by subsequent short periods of random or spontaneous synaptic activity (Zhou et al., 2003). These findings indicate that there are cellular memory processes that integrate over longer time periods (Fusi et al., 2005), consolidating and extending the duration of changes in synaptic strength. The identities of such processes remain to be determined, although some molecular candidates are starting to be determined (Pagani et al., 2009).

Long-term potentiation and LTD are processes that saturate (Levy and Steward, 1979; Rioult-Pedotti et al., 2000; Scharfman and Sarvey, 1985). In most studies of STDP, synaptic weight can be increased up to $200-300 \%$ or decreased down to $50 \%$ of the original size (Figures 2 and 4), but physiological changes beyond these limits are rarely reported. The factors that control the maximum and minimum values of synaptic strength are still unknown, although limitations in receptor phosphorylation status (Lee et al., 2000), postsynaptic density size (Rasse et al., 2005), and vesicular release (Zucker and Regehr, 2002) may each contribute to saturation of synaptic modification. In phenomenological models, however, it is straightforward to implement saturation as a fixed bound on net synaptic modification. For example, the total amount of LTP induced by pre $\rightarrow$ post pairs and the total amount of LTD induced by post $\rightarrow$ pre pairs can be independently calculated, and set equal to the boundary values if greater/less than the saturation levels (Sjöström et al., 2001; Froemke et al., 2006). Boundary values are somewhat arbitrary, but it is reasonable to set the saturation points to be the empirically-measured mean amount of LTP and LTD induced by 60 pre/post pairs at short spike time intervals (approximately 100\% increase for LTP and-50\% decrease for LTD). Incorporating saturation is necessary to prevent STDP models from producing unrealistic predictions of synaptic modification.

How are the effects of individual pre/post pairs combined to determine the net change in synaptic strength? There are two main ways of integrating the contributions of single pre/post pairs, additively or multiplicatively. The additive model is the linear sum of individual pairwise contributions: $\Delta w=\sum_{i, j} \Delta w_{i j}$, and the multiplicative model is the product of spike pair effects: $1+\Delta w=\Pi_{i, j}\left(1+\Delta w_{i j}\right)$, where $i$ and $j$ represent individual spikes of the pre- and postsynaptic activity patterns, respectively. This is similar to the question about how a single pre/post pair might change synaptic strength- either by an incremental increase or decrease of a fixed magnitude, or in proportion to the current weight (van Rossum et al., 2000; Rubin et al., 2001). The additive model is often used in STDP simulations (Song et al., 2000; Hopfield and Brody, 2004; Knoblauch and Sommer, 2003) and overall provides lowererror predictions than the multiplicative model (Froemke et al., 2006). However, LTP and LTD are usually reported as a percentage change in synaptic efficacy, and there is evidence that the amount of synaptic modification depends on initial synaptic strength (Bi and Poo, 1998; Debanne et al., 1999; van Rossum et al., 2000; Sjöström et al., 2001), suggesting that the net effect of spike pairing may be multiplicative, especially for LTD. Regardless of the algorithm, both the additive and multiplicative independent models of STDP, including saturating LTP and LTD processes, still fail to provide good predictions of the effects of complex spike trains (having individual prediction errors of 40-50\%). On average, predictions of the saturating independent model (Sjöström et al., 2001; Froemke and Dan, 2002; Froemke et al., 2006) are only weakly correlated with empirically-observed changes in synaptic strength $(r: \sim 0.1-0.2)$.

\section{FREQUENCY DEPENDENCE OF STDP}

Although these studies show that STDP can result from repetitive pairing of single pre- and postsynaptic action potentials (Bell et al., 1997; Bi and Poo, 1998; Feldman, 2000; Sjöström et al., 2001; Froemke and Dan, 2002; Campanac and Debanne, 2008; Zilberter et al., 2009), in practice STDP is often induced by several iterations of pre- and postsynaptic spike bursts consisting of a small number of action potentials triggered at a high rate (Debanne et al., 1996; Magee and Johnston, 1997; Markram et al., 1997; Debanne et al., 1998; Boettiger and Doupe, 2001; Sjöström et al., 2001; Karmarkar et al., 2002; Watanabe et al., 2002; Tzounopoulos et al., 2004; Froemke et al., 2006; Nevian and Sakmann, 2006; Shen et al., 2008). Markram et al. (1997) originally used high-frequency pre/post spike bursts $(10+\mathrm{Hz})$ to induce LTP between pairs of layer five cortical pyramidal neurons, as spike burst pairing at lower frequency did not affect synaptic strength. This dependence of STDP on pre/post spike frequency demonstrates that the independent model, in which pairwise contributions are linearly summed or multiplied together, cannot entirely account for the effects of spike trains more complex than single pairs repeated at a low inter-pair interval (roughly $<5 \mathrm{~Hz}$ ). Therefore, there should be history-dependent processes that govern STDP learning rules beyond pre/post spike intervals. These forms of higher-order temporal modulation are the subject of the remainder of this review.

For unitary connections between pairs of neocortical pyramidal cells in brain slices, trains of five pre/post pairs induced LTD when the postsynaptic spike train led the presynaptic train, but only for intratrain spike rates below $40 \mathrm{~Hz}$. At higher rates, the temporal precision seemed to break down, and LTP was induced regardless of the exact spike timing (Sjöström et al., 2001). At these connections LTP could not be induced with appropriately timed spike pairs unless the intratrain spike rate was at least 10-20 Hz (Markram et al., 1997; Sjöström et al., 2001). In an elegant study, Sjöström et al. (2001) showed that spike-timing-dependent LTP could be induced with low-frequency $(0.1 \mathrm{~Hz})$ repetition of single pre/post pairs, when extracellular stimulation was used to evoke EPSPs in the postsynaptic cell, rather than via direct stimulation of a single presynaptic neuron.

Most synapses show a similar breakdown of timing dependence and conversion from LTD to LTP when high-frequency trains are used to induce synaptic modification (Figure 5), although the number of spikes and frequency required for LTP vary between synapses (Sjöström et al., 2001; Froemke et al., 2006; Wittenberg and Wang, 2006). This rate-dependence and shift to LTP induction is reminiscent of the BCM model of synaptic plasticity, suggesting that these phenomena share a common set of underlying cellular mechanisms, e.g., $\mathrm{Ca}^{2+}$ influx through NMDA receptors (Zucker, 1999; Froemke et al., 2005; Feldman, 2009).

\section{STDP INDUCED WITH SPIKE TRIPLETS AND QUADRUPLETS}

To begin to predict the synaptic effects of complex spike trains, we have taken an incremental approach towards characterizing the history dependence of hippocampal and neocortical STDP. A 

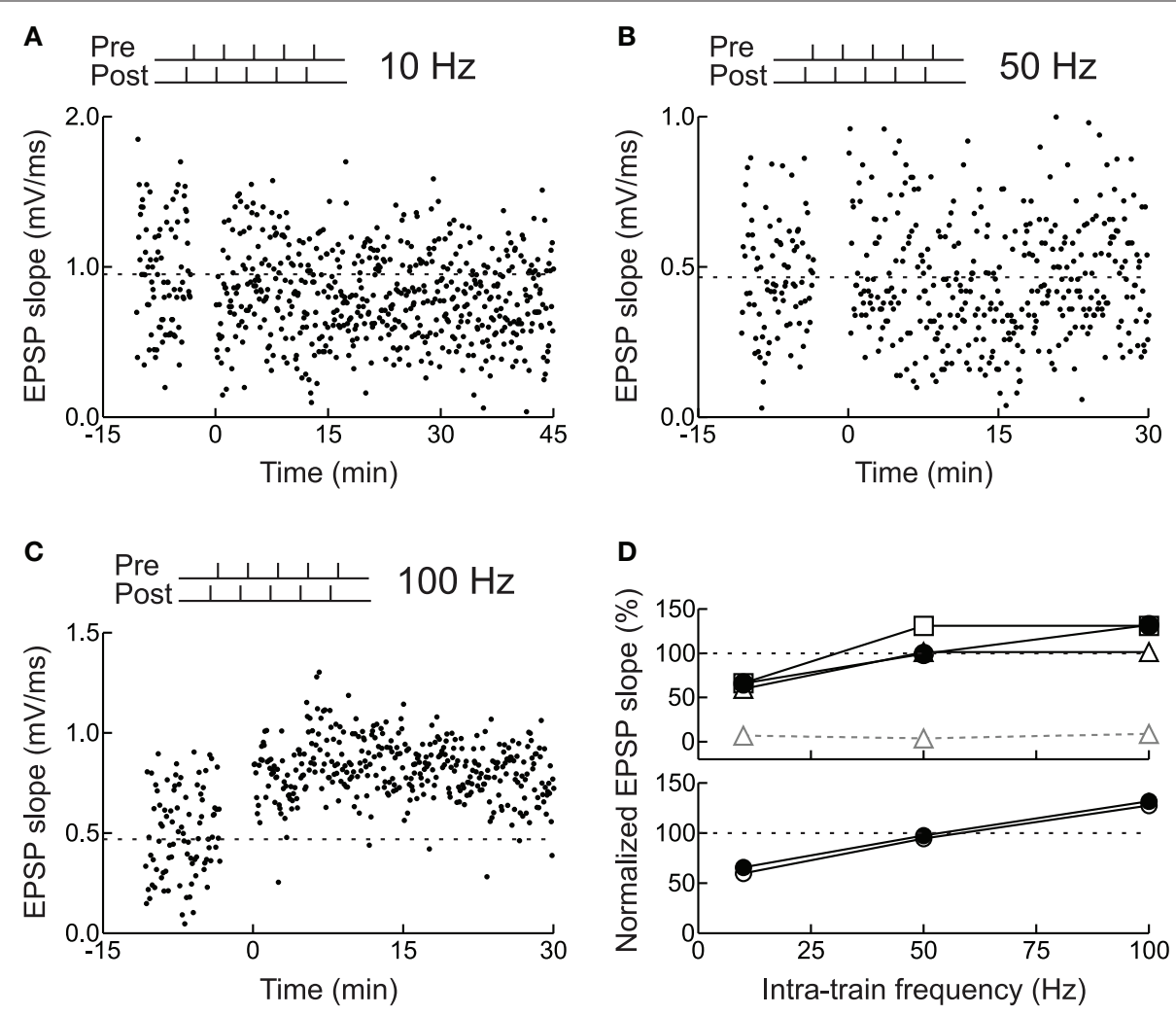

FIGURE 5 | Frequency dependence of STDP for layer $2 / 3$ neurons in acute slices of young rat visual cortex. (A) Example of LTD induced by pairing preand postsynaptic spike bursts. Each burst consisted of five spikes with an intra-train frequency of $10 \mathrm{~Hz}$ (i.e., inter-spike interval of $100 \mathrm{~ms}$ ). The postsynaptic train led the presynaptic train by $3 \mathrm{~ms}$. (B) As in (A), but no synaptic modification was induced when the intra-train frequency was $50 \mathrm{~Hz}$. (C) As in (A), but LTP was

straightforward way to study more complex spike trains is to systematically vary the parameters known to be important for induction of synaptic modification: the number, frequency, and precise timing of pre/post spikes presented during the induction protocol. In these experiments, we gradually increased the complexity of the pre/post spike pattern used to induce synaptic modification, starting by adding one additional spike -i.e., using spike triplets instead of spike pairs to induce long-term synaptic modification. From there, we have examined spike quadruplets and more complex burst patterns containing 6-12 action potentials at various inter-spike intervals (Debanne et al., 1994; Bi and Poo, 1998; Sourdet and Debanne, 1999; Froemke and Dan, 2002; Wang et al., 2005; Froemke et al., 2006). The goal of these parametric studies is the development of a phenomenological model, accounting for the rules and temporal modulation of STDP.

To determine the net change in synaptic strength by a given spike train, one possibility is that the effect of each pre/post spike pair is unaffected by the presence of other pre- and postsynaptic events. In this case, all pre/post pairs would be combined independently (either additively or multiplicatively), just by looking up each pre/post interval in the STDP time window (Figures 1 and 2). Alternatively, the presence of other spikes might somehow influence the contribution or eligibility of a given spike or spike pair for total synaptic modification. induced when the intra-train frequency was $100 \mathrm{~Hz}$. (D) Actual and predicted synaptic modification after burst pairing. Filled symbols, experiments. Open symbols, model predictions: (top) gray triangles, multiplicative independent model without saturation; black triangles, multiplicative model with saturation; squares, additive model with saturation; (bottom) circles, additive suppression model with saturation. From Froemke et al. (2006).
We tested this hypothesis by using spike triplets instead of spike pairs for STDP induction. Although there are eight $\left(2^{3}\right)$ basic ways to arrange three spikes among two neurons, two of these triplets are most informative for detecting history-dependent modulation of STDP: two presynaptic spikes flanking a single postsynaptic spike (pre $\rightarrow$ post $\rightarrow$ pre triplets) or two postsynaptic spikes flanking a presynaptic spike (post $\rightarrow$ pre $\rightarrow$ post triplets). In neurons from low-density hippocampal cultures, triplet experiments showed that post $\rightarrow$ pre $\rightarrow$ post triplets induced LTP, while pre $\rightarrow$ post $\rightarrow$ pre triplets led to no net change in synaptic strength (Bi and Poo, 1998; Bi and Wang, 2002; Wang et al., 2005; Figure 6A). This occurred even when the pre/post spike intervals were chosen to favor LTD, i.e., potentiation was dominant for these connections when the post $\rightarrow$ pre interval was shorter than the pre $\rightarrow$ post interval. Thus the effects of individual spike pairs in a triplet do not sum linearly - rather, LTP can cancel or "veto" previously induced LTD.

Almost the opposite effects were observed in layer $2 / 3$ lateral connections of visual cortex. For these synapses, post $\rightarrow$ pre $\rightarrow$ post triplets induced LTD, while pre $\rightarrow$ post $\rightarrow$ pre triplets induced LTP, even when the time interval of the second pre/post pairing was considerably shorter than the first interval (Froemke and Dan, 2002; Froemke et al., 2006; Figure 6B). Therefore the presence of the first spike or spike pair somehow suppressed the efficacy or eligibility of the last 

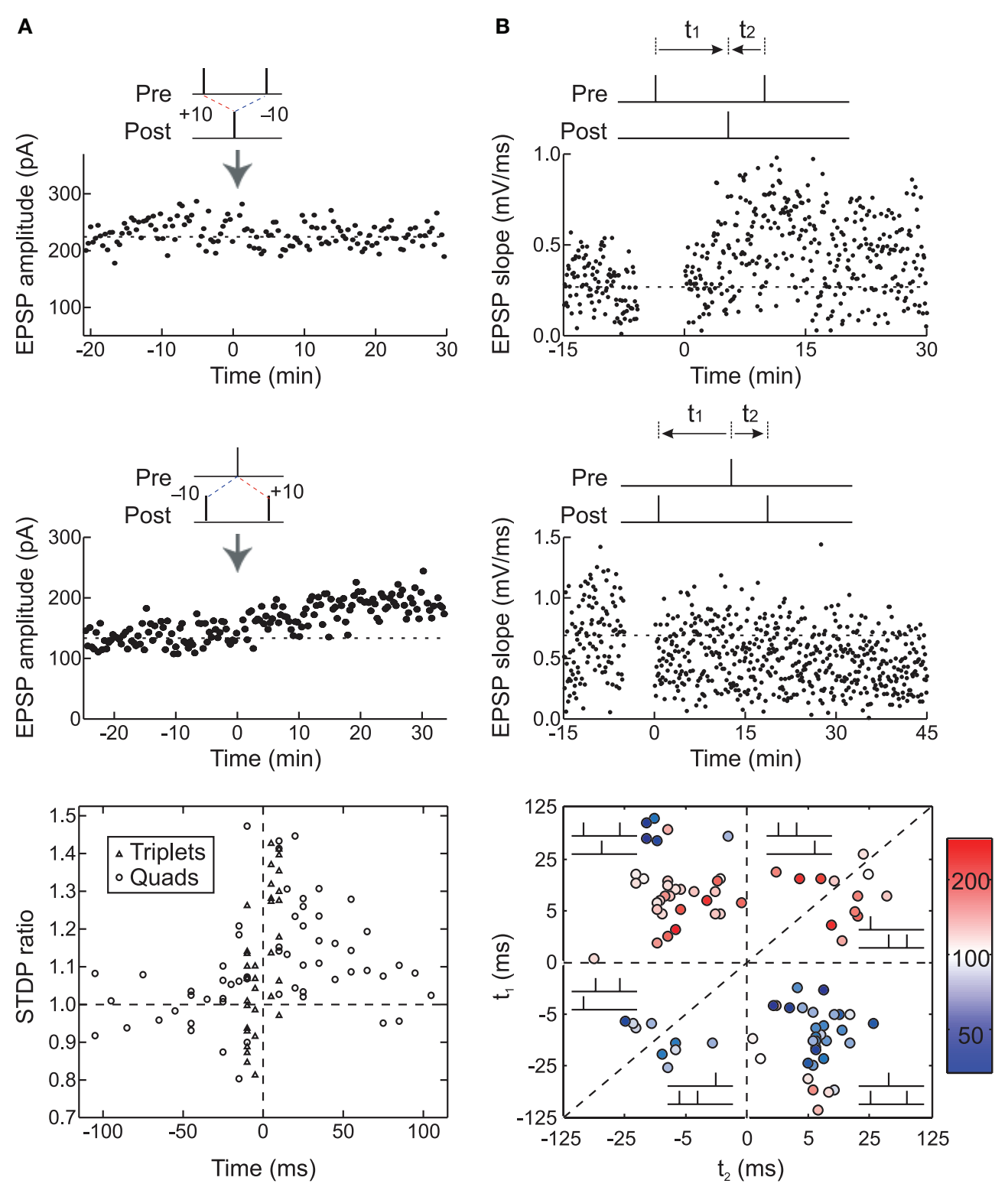

FIGURE 6 | Long-term synaptic modification induced by spike triplets. (A) In dissociated hippocampal cultured neurons, pre $\rightarrow$ post $\rightarrow$ pre triplets did not change synaptic strength (top) and post $\rightarrow$ pre $\rightarrow$ post triplets induced LTP (center). The time window for these temporal nonlinearities is $\sim 70 \mathrm{~ms}$; outside this interval, the effects of pre/post pairings were independent (bottom). From Wang et al. (2005). (B) In layer 2/3 visual cortical neurons in acute slices,

spike or spike pair to contribute to STDP. In other words, the effect of the first pair in the triplet was dominant for synaptic modification. These results are similar to the effects of pairing pre- and postsynaptic spike bursts in neurons of zebra finch forebrain - regardless of the timing of subsequent spikes, if a pre $\rightarrow$ post pair began the train, LTP was induced, but if the postsynaptic cell fires the first spike, LTD was induced (Boettiger and Doupe, 2001).

The nonlinear effects of spike triplets predicted the effects of spike quadruplets on STDP induction for both hippocampal and neocortical synapses. We used two kinds of quadruplets, pre $\rightarrow$ post $\rightarrow$ pre triplets induced LTP (top) and post $\rightarrow$ pre $\rightarrow$ post triplets induced LTD (center), demonstrating that the first spike pair is dominant in STDP, and the effect of the second spike pair is suppressed by the first. The time window for presynaptic suppression was found to be shorter than the duration of postsynaptic suppression (bottom). From Froemke and Dan (2002), and Froemke et al. (2006). pre $\rightarrow$ post $\rightarrow$ post $\rightarrow$ pre (type A: a pre $\rightarrow$ post pair followed at a short interval by a post $\rightarrow$ pre pair) and post $\rightarrow$ pre $\rightarrow$ pre $\rightarrow$ post (type B: a post $\rightarrow$ pre pair followed at a short interval by a pre $\rightarrow$ post pair). For hippocampal neurons, type A quadruplets did not affect synaptic strength (similar to pre $\rightarrow$ post $\rightarrow$ pre triplets), and type B quadruplets induced LTP (similar to post $\rightarrow$ pre $\rightarrow$ post triplets) (Wang et al., 2005). Conversely, for cortical neurons (Froemke and Dan, 2002), type A quadruplets induced LTP and type B quadruplets induced LTD, due to suppression of the effects of the latter spikes in the sequence, but only when the inter-pair interval (the post $\rightarrow$ post 
interval for type A or the pre $\rightarrow$ pre interval for type B) was relatively short (approximately $<100-200 \mathrm{~ms}$ ). For longer inter-pair intervals, the effects of the two pairs summed linearly.

The effects of pre/post spike pairs are almost identical for hippocampal and neocortical neurons (Figures 1 and 2). Why then would spike triplets and quadruplets operate so differently for these two systems (Figure 6)? We hypothesize that there are distinct processes that govern the temporal modulation of STDP, controlling the eligibility of a given spike for contributing to synaptic modification under the pairwise STDP learning rule.

One set of important phenomena that could modulate the effectiveness of presynaptic spike trains for STDP are forms of short-term synaptic plasticity such as paired-pulse depression (PPD) or pairedpulse facilitation (PPF). When a presynaptic neuron fires twice within about $200 \mathrm{~ms}(5+\mathrm{Hz})$, the amplitude of the second event is often facilitated (for PPF) or depressed (for PPD) relative to the size of the first event (Zucker and Regehr, 2002). These forms of short-term plasticity have the capability to influence the induction of long-term plasticity because NMDA receptor activation and consequently, the level of postsynaptic $\mathrm{Ca}^{2+}$ influx, depend on the amount of presynaptic transmitter release. In the limit, if a synapse shows strong PPD, such that the amount of transmitter released by the second presynaptic spike is essentially zero, then in terms of NMDA receptor activation and STDP, it is as if the second spike never occurred (i.e., pre $\rightarrow$ post $\rightarrow$ pre triplets would be equivalent to pre $\rightarrow$ post pairs). Conversely, if a synapse is strongly facilitating, evoking little to no EPSP from the first presynaptic spike but with EPSPs growing in size with subsequent spikes, the second presynaptic spike will be much more important than the first for determination of net synaptic modification (i.e., in this case a pre $\rightarrow$ post $\rightarrow$ pre triplet would be equivalent to a post $\rightarrow$ pre pair). Therefore, given that most synapses show some form of short-term plasticity (Zucker and Regehr, 2002), it would be surprising if the effect of spike triplets in STDP was just the linear sum of the pairwise contributions. By similar logic, temporal modulation of STDP may be different between synapses that facilitate and synapses that depress, at least for the effects of presynaptic spikes in a burst within the time scale of short-term plasticity.

In agreement with this idea, excitatory neocortical synapses usually exhibit PPD, especially during development (Reyes and Sakmann, 1999; Froemke et al., 2006), while PPF can often be observed at hippocampal synapses (Buonomano, 1999; Poncer and Malinow, 2001), although is not pronounced for cultured neurons (Wang et al., 2005). This fundamental difference in synaptic transmission might at least partially account for the dissimilar effects of spike triplets for STDP induction in these preparations. Under this hypothesis, pre $\rightarrow$ post $\rightarrow$ pre triplets at cortical synapses induce LTP due to suppression of the effect of the post $\rightarrow$ pre pair via presynaptic PPD. Conversely, in cultured hippocampal neurons, these triplets would not affect synaptic strength as the efficacy of the post $\rightarrow$ pre pair would also have a significant effect. Additional support for the role of short-term plasticity in temporal modulation of STDP comes from pharmacological experiments in visual cortical slices. Increasing PPD led to enhanced presynaptic suppression of STDP, while converting PPD into PPF removed presynaptic suppression, increasing the independence of these spikes and essentially linearizing the effects of presynaptic bursts for long-term synaptic modification (Froemke et al., 2006).
In a similar manner, postsynaptic spike bursts might modulate STDP induction through regulation of postsynaptic excitability or changes in presynaptic transmitter release. Both types of modulation have been found for hippocampal and neocortical synapses, implemented through a wide range of cellular mechanisms. Some of the best characterized activity-dependent regulators of postsynaptic excitability are $\mathrm{K}^{+}$channels (Debanne et al., 1997; Hoffman et al., 1997). In response to an initial increase in membrane potential, $\mathrm{K}^{+}$channel activation limits further depolarization, potentially weakening the impact of subsequent postsynaptic spikes for STDP induction. During high-frequency trains of postsynaptic spiking, action potentials can be observed to attenuate in size such that latter spikes in a burst are smaller than earlier spikes, which might impact gating of postsynaptic NMDA receptors and STDP induction (Tanaka et al., 1991; Froemke et al., 2006). Several groups have used pharmacological or genetic manipulation of A-type $\mathrm{K}^{+}$ channels and SK channels in hippocampal and cortical neurons to show that these ion channels raise the threshold and enforce spike timing precision of STDP (Watanabe et al., 2002; Chen et al., 2006; Froemke et al., 2006; Lin et al., 2008). For visual cortical synapses, blockade of A-type channels widened the time window for timingdependent LTD (Froemke et al., 2005), and removed postsynaptic suppression, such that post $\rightarrow$ pre $\rightarrow$ post triplets induced LTP instead of LTD (Froemke et al., 2006). This result, similar to the dominating effects of the LTP-inducing pair in post $\rightarrow$ pre $\rightarrow$ post triplets in hippocampal cultures, could indicate additional nonlinearity in downstream signal integration (Bi and Rubin, 2005; Wang et al. 2005).

At some synapses, prolonged postsynaptic depolarization leads to release of endocannabinoids, which act as retrograde messengers to decrease transmitter release and induce spike-timing-dependent LTD (Sjöström et al., 2003; Bender et al., 2006; Tzounopoulos et al., 2007). Furthermore, postsynaptic bursting by layer 5 neurons widens the time window for LTD induction (Sjöström et al., 2003), but postsynaptic depolarization in absence of spike firing is sufficient to induce LTD in those cells (Sjöström et al., 2004). At synapses onto layer $2 / 3$ cells activated by focal extracellular stimulation near the apical dendrite, however, postsynaptic depolarization causes $\mathrm{Ca}^{2+}$ dependent suppression of NMDA receptor activation. Potentially as a direct consequence of reduced $\mathrm{Ca}^{2+}$ flux through NMDA receptor channels, post $\rightarrow$ pre pairing induces LTD (Froemke et al., 2005; Urakubo et al., 2008). In both cases, the activity of the postsynaptic neuron seems to determine the timing requirements for LTD, suggesting that longer periods of postsynaptic spiking should prolong this temporal window. Indeed, this seems to be the case- for hippocampal neurons, the LTD time window is controlled by the exact number of spikes during a postsynaptic burst. When a single postsynaptic spike preceded presynaptic activation, LTD was induced only when the post $\rightarrow$ pre time interval was $<200 \mathrm{~ms}$. However, when a burst of four postsynaptic spikes was used instead, this interval was extended up to $800 \mathrm{~ms}$, and a burst of ten spikes extended this window further, to $2000 \mathrm{~ms}$ (Debanne et al., 1994; Sourdet and Debanne, 1999). Together, these results suggest that it should be possible to build quantitative models that accurately predict, spike by spike, the net change in synaptic strength induced by a given spike train. The tight linkage between specific cellular mechanisms and aspects of STDP induction and temporal modulation further 
suggests that biologically realistic simulations could eventually be used to predict how complex spike trains might induce enduring synaptic modifications.

\section{PREDICTING THE EFFECTS OF COMPLEX SPIKE TRAINS}

Complex spike trains with irregular patterns of spiking are not usually used as induction protocols for synaptic plasticity (Paulsen and Sejnowski, 2000). A seminal paper by Dobrunz and Stevens (1999) was the first study to use natural spike trains to induce long-term synaptic modification in slices of rat hippocampus. While that study demonstrated that repetitive presentation of natural spike train segments could lead to LTP, no attempt was made to predict or quantify the impact of individual spike trains on synaptic plasticity. Sjöström et al. (2001) then used random sequences of pre/post spike trains at various intra-train frequencies to induce synaptic modification. They used an additive model in which postsynaptic depolarization determined the amount of LTP, only nearest-neighbor spike pairs contributed to STDP, and spikes that participated in an LTP pairing could not participate in an LTD pairing (such that LTP effectively "wins out" over LTD). Their model captured the essential frequency dependence of STDP for cell pairs and predicted the net synaptic modification induced by random trains with high accuracy.

In slices of the mammalian lateral geniculate nucleus (LGN), stimulation of optic tract afferents onto thalamic neurons induced LTP or LTD depending on the time differences of pre- and postsynaptic spike bursts (Butts et al., 2007). Spike bursts consisted of 10-20 Hz trains for one second, simulating natural activity patterns that occur during spontaneous retinal waves. LTP was induced when the latency between bursts occurred within about $500 \mathrm{~ms}$, regardless of the pre/post temporal ordering. The independent model of STDP failed to predict the extent of synaptic modification induced by burst pairing, but as in Sjöström et al. (2001), modifying STDP to consider only nearest-neighbor spikes and removing LTD significantly improved predictions of the model.

We wondered if a similar approach could be used more generally to account for the effect of sparse and irregular spike trains in layer $2 / 3$ cortical connections. The aim of these experiments was to use STDP to quantitatively describe how the fine temporal structure within complex and naturalistic spike trains would influence the sign and magnitude of overall long-term synaptic modification, solely at the level of individual spikes. Specifically, we set out to construct an accretive, low-parameter phenomenological model that captured most of the variance in STDP induced by a wide range of different spiking patterns: spike pairs, triplets, quadruplets, lowand high-frequency bursts, and natural spike train fragments.

We started by extending the history-independent model of STDP to account for the effects of spike triplets. In this model (the "suppression" model), the contribution of a pre/post spike pair depends not only on the time interval between the two spikes (Figure 2), but also on the efficacy of each spike (Froemke and Dan, 2002). This spike efficacy is the eligibility of a spike to participate in synaptic modification, and acts to scale down the effect of a pre/post pair. Spike efficacy is defined as a coefficient between zero and one depending on the time from the preceding spike: it begins at one (full strength), but is suppressed to zero immediately after a spike, and recovers exponentially back to one. To predict the effects of spike train segments using the suppression model, each pre- and postsynaptic spike was assigned an efficacy, which depends only on the interval from the preceding spike in the same neuron: $\varepsilon_{i}=1-e^{-\left(t_{i}-t_{i-1}\right) / \tau_{s}}$. Here, $\varepsilon_{\mathrm{i}}$ is the efficacy of the $i$ th spike, $t_{i}$ and $t_{i-1}$, are the timing of the $i$ th and $(i-1)$ th spike, respectively, and $\tau_{s}$, is the time constant of single exponential recovery from suppression. Suppression time constants for the pre- and postsynaptic neurons, $\tau_{\mathrm{s}}^{\text {pre }}$ and $\tau_{\mathrm{s}}^{\text {post }}$, were determined from the $2-1$ and 1-2 triplet experiments (Figure 6B), chosen to minimize mean prediction error (|predicted effect - measured effect $\mid)$. The contribution of each pre/post spike pair to synaptic modification was estimated as $\Delta w_{i j}=\varepsilon_{i}^{\text {pre }} \varepsilon_{j}^{\text {post }} F\left(\Delta t_{i j}\right)\left(\Delta \mathrm{w}_{\mathrm{ij}}\right.$, synaptic modification due to the $i$ th presynaptic spike and the $j$ th postsynaptic spike; $\varepsilon_{i}^{\text {pre }}$ and $\varepsilon_{j}{ }^{\text {post }}$, efficacy of the two spikes, respectively; $\Delta t_{i j}$, the interval between the two spikes, $\left.t_{j}^{\text {post }}-t_{i}^{\text {pre }}\right)$. The function $F$ represents the temporal window for STDP measured with isolated spike pairs, expressed as:

$F(\Delta t)=\left\{\begin{array}{cc}A_{+} e^{-|\Delta t| / \tau_{+}} & \text {if } \Delta t>0 \\ A_{-} e^{-|\Delta t| / \tau_{-}} & \text {if } \Delta t<0\end{array}\right.$

( $A$, scaling factor; $\tau$, time constant; +, LTP; -, LTD). The historyindependent model is then a special case of the suppression model, where spike efficacies $\varepsilon_{\mathrm{i}}$ are always 1 for all $i$ regardless of interspike interval.

Both the additive and multiplicative versions of the suppression model, without saturation, made significantly more accurate predictions than did the history-independent models of STDP induced by spike triplets, quadruplets, and fragments of natural spike trains initially recorded in vivo (Froemke and Dan, 2002). The suppression model prediction errors for individual cases were approximately $15-30 \%$, compared to the larger errors of $30-50 \%$ produced by the history-independent model. These values are close to the individual error intrinsic to the single exponential fits to the pre/post spike pair experiments themselves ( $15 \%)$, which can be considered the limit of predictive power for any model based on the pairwise STDP learning rule.

Thus, the original suppression model provided a good description of STDP induced by complex spike patterns encountered in vivo (Froemke and Dan, 2002). However, as this model was derived from spike pair and triplet experiments where temporal precision was a key parameter, it was not designed to account for the breakdown of STDP and rate-dependent conversion to LTP when high frequency bursts of five presynaptic and five postsynaptic spikes ("5-5" trains) were used for induction (Markram et al., 1997; Sjöström et al., 2001). Various reformulations of the original suppression model - with or without saturation of STDP, and assuming either additive or multiplicative combinations (open symbols in Figure 5D, top) - also failed to entirely account for the frequency dependence of STDP, although including saturating LTP and LTD into the suppression model greatly reduced prediction errors on high-frequency bursts (compare gray triangles with dashed line to triangles and squares with solid lines in Figure 5D, top). Therefore we tried to identify the processes that contribute to inter-spike suppression and frequency-dependent potentiation, and determine what additional factors or parameters beyond saturation were required for a more complete model (the "revised suppression" model of STDP). 
We systematically varied the number of spikes between one and five in a high-frequency burst $(100 \mathrm{~Hz})$ of either the pre- or postsynaptic train, fixing the number of spikes in the other train to be one (" $x-1$ " or " $1-x$ " trains); in both cases the leading pre/post pair was post $\rightarrow$ pre (Froemke et al., 2006). We first examined the contribution of postsynaptic spiking. Using 1-5 trains for induction induced LTP; surprisingly, this effect was much more consistent with the history-independent model than with the suppression model. In general, there was a conversion from LTD to LTP as the number of postsynaptic spikes was increased over a range of one to five. The degree of potentiation and the gradual shift from LTD to LTP with an increasing number of postsynaptic spikes were strikingly similar to that observed in the 5-5 train experiments, strongly suggesting that the number and frequency of postsynaptic spikes, rather than presynaptic spikes, are main determinants of frequencydependent STDP. This finding is consistent with earlier results from Gustafsson et al. (1987) and Debanne et al. (1994), showing that the number of postsynaptic spikes in a burst was correlated with the amount of LTP, and agree with work from Pike et al. (1999), in which postsynaptic but not presynaptic bursting was predominant for LTP induction. All of these experiments are reminiscent of the classic BCM model of long-term synaptic plasticity (Bienenstock et al., 1982), in which the level of postsynaptic activity is critical for determining the sign and magnitude of synaptic modification.

How might these results be reconciled with the original suppression model of STDP? One possibility is that a burst of postsynaptic spikes has certain synergy, and is qualitatively different from the individual component spikes within the burst. In this scheme, high-frequency postsynaptic bursting would tend to induce LTP whenever temporally correlated with presynaptic activity, irrespective of the exact spike timing. This is similar to the suggestion of Sjöström et al. (2001) for cortical layer 5 synapses, in which LTP is dominant over LTD when considering nearest-neighbor interactions. To test this hypothesis for layer $2 / 3$ connections, we examined an alternate version of the 1-5 spike train, in which the single presynaptic spike has been moved towards the end of the train, occurring between the fourth and fifth postsynaptic spikes. Despite the high-frequency burst of five postsynaptic spikes, this protocol induced LTD (Froemke et al., 2006). Thus for layer 2/3 synapses in developing visual cortex, high-frequency bursts do not always induce LTP, and instead the sign and amount of synaptic modification still depends on the precise timing and arrangement of pre/post spikes.

In these experiments, there appeared to be a gradual relaxation of postsynaptic suppression, with the suppression model accounting for 1-2 trains (i.e., post $\rightarrow$ pre $\rightarrow$ post triplets) and the history-independent model accounting for 1-5 trains (i.e., post $\rightarrow$ pre $\rightarrow$ post $\rightarrow$ post $\rightarrow$ post $\rightarrow$ post bursts $)$. Therefore, we altered the suppression model such that postsynaptic inter-spike suppression depended not only on the time from the previous postsynaptic spike, but also depended inversely on the degree of suppression of that spike. This can be formalized as: $\varepsilon_{i}=1-\varepsilon_{i-1} \times e^{-\left(t_{i}-t_{i-1}\right) / \tau_{s}}$, where $\varepsilon_{i}$ is the efficacy of the $i^{\text {th }}$ postsynaptic spike (with the first postsynaptic spike having $\varepsilon_{i}=1$ ). Incorporating this change made the suppression model behave as the history-independent model for 5-5 trains, suggesting that the revisions to the suppression model were not yet entirely complete.
We then examined the effect of presynaptic bursting. We found that $x-1$ trains, with a single postsynaptic spike and a variable number of presynaptic spikes, induced LTD in all cases. Given the high degree of short-term depression and the long time course of NMDA receptor activation at these synapses, it is perhaps not surprising that all $x-1$ spike trains produce approximately the same amount of depression. In other words, increasing the number of presynaptic spikes has little direct effect on the degree of synaptic modification induced by high-frequency spike trains. One interpretation of these results is that inter-spike suppression of presynaptic efficacy must also depend not only on the time to the previous spike, but also on the degree of suppression of this spike. Rather than depending inversely, presynaptic suppression may accumulate, in a manner reminiscent of short-term depression. We therefore corrected presynaptic efficacy to be: $\varepsilon_{i}=\Pi_{j=1}^{i}\left(1-e^{-\left(t_{i}-t_{i-j}\right) / \tau_{s}}\right)$, similar to the scheme used by Varela et al. (1997) for simulating neocortical short-term depression. In this scheme, the efficacy of each presynaptic spike depends on the time to all preceding spikes in the presynaptic burst. When this change was made to presynaptic suppression, the revised additive suppression model of STDP well-predicted the effects of high-frequency spike trains (Figure 5D, bottom).

Thus the suppression model was revised to account for the frequency-dependence of STDP by three modest corrections: one, a history-dependent relaxation of postsynaptic suppression; two, a history-dependent increase in presynaptic suppression; and three, incorporating physiological levels of saturation. Overall, the individual prediction error of the revised suppression model was approximately $10 \%$, significantly better than each of the other models. For individual intra-train frequencies, the revised suppression model correctly predicted the gradual shift from LTD to LTP. Comparing mean amounts of synaptic modification induced at different intra-train spike rates, as in Sjöström et al. (2001), gave the following prediction errors: $10 \mathrm{~Hz}, 6.2 \%$; $50 \mathrm{~Hz}, 2.8 \%$; and $100 \mathrm{~Hz}, 4.2 \%$. Additionally, the revised suppression model accounted for the results of the $x$ - 1 and 1- $x$ experiments, quadruplets, and natural spike train fragments, resulting in a high correlation between predicted and empirically measured synaptic modification ( $r: 0.6-0.8$; Figures $5 \mathrm{D}$ and 7$)$. The prediction errors of this model over all such complex spike trains (10-20\% individual prediction errors) were comparable to the errors of the original suppression model tested only on the relatively sparse natural spike trains, and also comparable to the error inherent in the critical time window for spike pairs. It is difficult to improve the predictions of the models beyond errors of $\sim 15 \%$ for individual experiments. This degree of error is also evident in the fits to the temporal window for pre/post spike pairs, and perhaps reflects heterogeneity among cells, e.g., an inherent bias toward LTP or LTD that may depend on how close the baseline synaptic strength is toward saturation.

Of course, the revised suppression model accounts only for the effects of complex spike trains in layer $2 / 3$ cortical neurons. Given the difference in results between Figures $6 \mathrm{~A}$ and $6 \mathrm{~B}$, this model cannot account per se for the effects of spike triplets and quadruplets in cultured hippocampal neurons. In particular, post $\rightarrow$ pre $\rightarrow$ post triplets increased synaptic strength by $\sim 25-30 \%$ in cultured neurons (with either 5 or $10 \mathrm{~ms}$ inter-spike intervals) but decreased synaptic strength by about $-20 \%$ in layer $2 / 3$ of visual cortex, while pre $\rightarrow$ post $\rightarrow$ pre triplets did not affect synaptic strength in cultured 
A

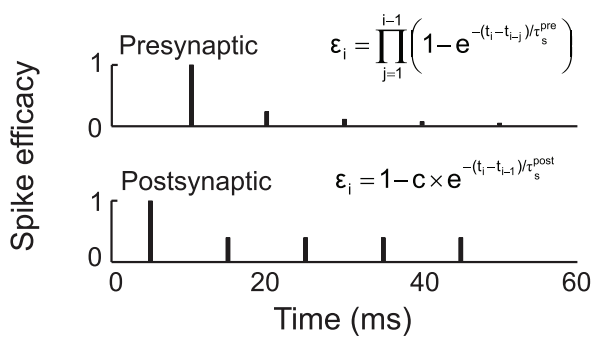

B

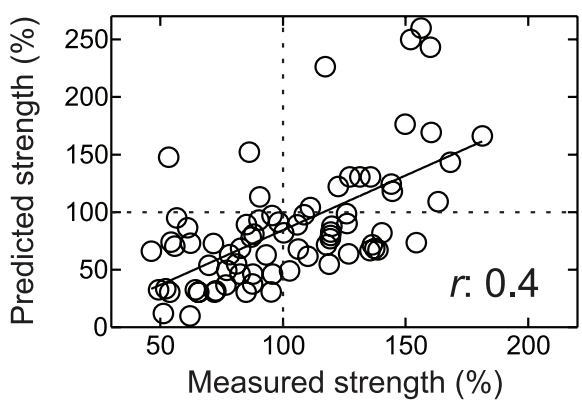

C

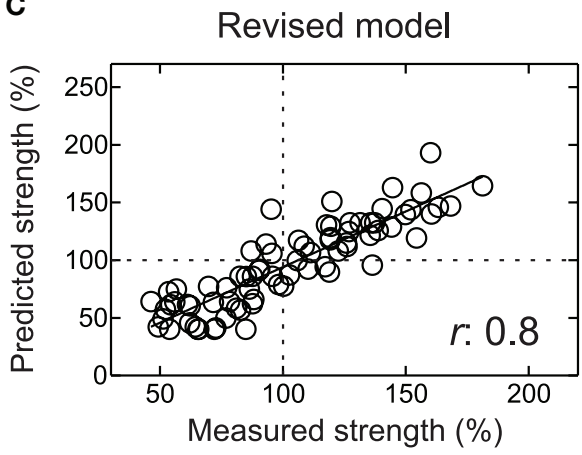

FIGURE 7 |The revised suppression model of STDP predicts the effects of complex and naturalistic spike trains. (A) Schematic of the revised suppression model. The height of each spike represents the efficacy of that event for contributing to STDP. (B) Comparison of predicted and measured effects of complex spike trains (quadruplets, high-frequency bursts, and natural spike train fragments) used to induce synaptic modification. Predicted values are from the additive history-independent model, with saturation of LTP and LTD and the number of pre/post spike pair repetitions taken into consideration. Each circle indicates one experiment. The linear correlation coefficient $r$ between predicted and measured amount of STDP was 0.4. (C) As in (B), but with using the predictions of the revised suppression model. The linear correlation coefficient was 0.8. From Froemke et al. (2006).

cells but enhanced EPSPs by $\sim 25 \%$ in cortex (Froemke and Dan, 2002; Wang et al., 2005). However, short-term plasticity is but one way in which temporal modulation of STDP multi-spike interactions could be implemented in biological networks. For cultured hippocampal neurons, the nonlinear dynamics of biochemical activity (e.g., kinase and phosphatase metabolism) may be predominant over the contributions of other factors such as pairedpulse depression or facilitation (Rubin et al., 2005). Regardless, it may be possible to capture the effects of these interactions in a phenomenological framework like the suppression model of STDP, using the results of mechanistic experiments to inform the choice of variables and guide exploration through parameter space. We also note that the suppression model can recapitulate major features of STDP at other synapses with relatively straightforward changes- reducing presynaptic suppression mimics the effects of pre $\rightarrow$ post $\rightarrow$ pre triplets in cultured hippocampal neurons, while reducing postsynaptic suppression captures the lower-frequency shift to LTP with trains of pre- and postsynaptic spikes as observed for pairs of layer five pyramidal cells by Sjöström et al. (2001).

\section{CONCLUSION}

Determining the rules of long-term synaptic modification is crucial to understanding brain function and development, and several attempts have been made to build computational models of phenomena such as LTP and LTD. One of the most successful has been the BCM model, a rate-based approach developed in the 1980s toward understanding how synaptic modifications might underlie functional rearrangement of the cortex. However, work from many laboratories over the last 15 years has shown that the precise timing of spikes in the pre- and postsynaptic neurons is the critical determinant of long-term synaptic plasticity, likely due to the nonlinear dependence of NMDA receptor activation and postsynaptic $\mathrm{Ca}^{2+}$ influx on membrane potential. For several reasons, STDP has become a standard protocol for both experimental and theoretical investigation of learning and memory, and the STDP learning rule can be used to make accurate predictive models of the sign and magnitude of long-term synaptic modification induced by complex and naturalistic spike trains.

Collectively, these experiments are a proof-of-concept demonstration that STDP provides a basis for constructing such models. Here we have concentrated on characterizing history-dependent temporal nonlinearities that occur on relatively short timescales, from milliseconds to minutes. Forms of temporal modulation over longer periods, such as metaplasticity or homeostatic synaptic scaling, also play important roles in adjusting synaptic strength and organizing neural circuits (Abraham and Bear, 1996; Turrigiano and Nelson, 2000). Other types of modulation related to reinforcement schedule, arousal level, and motivational state (Cruikshank and Weinberger, 1996; Buonomano and Merzenich, 1998; Martin et al., 2000; Froemke et al., 2007; Zhang et al., 2009) will also eventually need to be quantified and incorporated into these models. The development of such hybrid phenomenological models incorporating mechanistic elements may be promising for providing better predictions of the effects of certain forms of experience on synaptic transmission. In the end, large-scale simulations of neural processing, with realistic forms of synaptic plasticity, learning, and memory, will be essential for the creation and optimization of behavioral programs, drugs, and devices for the treatment of neuropsychiatric disorders (Markram, 2006).

\section{ACKNOWLEDGMENTS}

The experiments on STDP of layer 2/3 cortical pyramidal cells were performed in the laboratory of Yang Dan. We are indebted to Beat Gähwiler and Scott Thompson for the help with experiments on STDP of CA3 pyramidal neurons. This work was supported by grants from the NEI, NIDCD, and the Howard Hughes Medical Institute (Robert C. Froemke); and grants from INSERM, CNRS, ANR and Région PACA (Dominique Debanne). 


\section{REFERENCES}

Abarbanel, H. D. I., Huerta, R., and Rabinovich, M. I. (2002). Dynamical model of long-term synaptic plasticity. Proc. Natl. Acad. Sci. U.S.A. 99, 10132-10137.

Abbott, L. F., and Blum, K. I. (1996). Functional significance of long-term potentiation for sequence learning and prediction. Cereb. Cortex 6, 406-416.

Abbott, L. F., and Nelson, S. B. (2000). Synaptic plasticity: taming the beast. Nat. Neurosci. 3, 1178-1183.

Abraham, W. C., and Bear, M. F. (1996). Metaplasticity: the plasticity of synaptic plasticity. Trends Neurosci. 19, 126-130.

Artola, A., Bröcher, S., and Singer, W. (1990). Different voltage-dependent thresholds for inducing long-term depression and long-term potentiation in slices of rat visual cortex. Nature 347, 69-72.

Baranyi,A., and Fehér, O. (1981). Synaptic facilitation requires paired activation of convergent pathways in the neocortex. Nature 290, 413-415.

Bauer, E. P., LeDoux, J. E., and Nader, K. (2001). Fear conditioning and LTP in the lateral amygdala are sensitive to the same stimulus contingencies. Nat. Neurosci. 4, 687-688.

Bell, C. C., Han, V. Z., Sugawara, Y., and Grant, K. (1997). Synaptic plasticity in a cerebellum-like structure depends on temporal order. Nature 387, 278-281.

Bender, V. A., Bender, K. J., Brasier, D. J., and Feldman, D. E. (2006). Two coincidence detectors for spike timingdependent plasticity in somatosensory cortex. J. Neurosci. 26, 4166-4177.

Berninger, B., and Bi, G.-Q. (2002). Synaptic modification in neural circuits: a timely action. Bioessays 24 212-222.

Bi, G.-Q., and Poo, M.-M. (1998). Synaptic modifications in cultured hippocampal neurons: dependence on spike timing, synaptic strength, and postsynaptic cell type. J. Neurosci. 18, 10464-10472.

Bi, G.-Q., and Poo, M.-M. (2001).Synaptic modification by correlated activity: Hebb's postulate revisited. Annu. Rev. Neurosci. 24, 139-166.

Bi, G.-Q., and Rubin, J. (2005). Timing in synaptic plasticity: from detection to integration. Trends Neurosci. 28, 222-228.

Bi, G.-Q., and Wang, H. X. (2002). Temporal asymmetry in spike timingdependent synaptic plasticity. Physiol. Behav. 77, 551-555.

Bienenstock, E. L., Cooper, L. N., and Munro, P. W. (1982). Theory for the development of neuron selectivity: orientation specificity and binocular interaction in visual cortex. J. Neurosci. 2, 32-48.

Bindman, L. J., Lippold, O. C., and Redfearn, J. W. (1962). Long-lasting changes in the level of the electrical activity of the cerebral cortex produced by polarizing currents. Nature 196, 584-585.

Birtoli, B., and Ulrich, D. (2004). Firing mode-dependent synaptic plasticity in rat neocortical pyramidal neurons. J. Neurosci. 24, 4935-4940

Bliss, T. V., and Lømo, T. (1973). Longlasting potentiation of synaptic transmission in the dentate area of the anesthetized rabbit following stimulation of the perforant path. $J$. Physiol. 232, 331-356.

Boettiger, C. A., and Doupe, A. J. (2001) Developmentally restricted synaptic plasticity in a songbird nucleus required for song learning. Neuron 31, 809-818.

Buchanan, K. A., and Mellor, J. R. (2007) The development of synaptic plasticity induction rules and the requirement for postsynaptic spikes in rat hippocampal CA1 pyramidal neurones. J. Physiol. 585, 429-445.

Buonomano, D. V. (1999). Distinct functional types of associative longterm potentiation in neocortical and hippocampal pyramidal neurons. J. Neurosci. 19, 6748-6754.

Buonomano, D.V., and Merzenich, M.M. (1998). Cortical plasticity: from synapses to maps. Annu. Rev. Neurosci. 21, 149-186.

Butts, D. A., Kanold, P. O., and Shatz, C. J. (2007). A burst-based "Hebbian" learning rule at retinogeniculate synapses links retinal waves to activitydependent refinement.PLoS Biol.5, e61. doi:10.1371/journal.pbio.0050061.

Campanac, E. and Debanne, D. (2008) Spike timing-dependent plasticity: a learning rule for dendritic integration in rat CA1 pyramidal neurons. J. Physiol. 586, 779-793.

Caporale, N., and Dan, Y. (2008). Spike timing-dependent plasticity: a Hebbian learning rule. Annu. Rev. Neurosci. 31, 25-46.

Chen, X., Yuan, L. L., Zhao, C., Birnbaum, S. G., Frick, A., Jung, W. E., Schwarz, T. L., Sweatt, J. D., and Johnston, D. (2006). Deletion of Kv4.2 gene eliminates dendritic A-type current and enhances induction of long-term potentiation in hippocampal CA1 pyramidal neurons. J. Neurosci. 26, 12143-12151.

Corlew, R., Wang, Y., Ghermazien, H., Erisir, A., and Philpot, B. D. (2007). Developmental switch in the contribution of presynaptic and postsynaptic NMDA receptors to long-term depression. J. Neurosci. 27, 9835-9845.
Cruikshank, S. J., and Weinberger, N M. (1996). Evidence for the Hebbian hypothesis in experience-dependent physiological plasticity of neocortex: a critical review. Brain Res. Brain Res. Rev. 22, 191-228.

Dan, Y., and Poo, M. M. (2006). Spike timing-dependent plasticity: from synapse to perception. Physiol. Rev. 86, 1033-1048.

Debanne, D., Gähwiler, B. H. and Thompson, S. M. (1994). Asynchronous pre- and postsynaptic activity induces associative long-term depression in area CA1 of the rat hippocampus in vitro. Proc. Natl. Acad. Sci. USA 91, 1148-1152.

Debanne, D., Gähwiler, B. H., and Thompson, S. M. (1996). Cooperative interactions in the induction of longterm potentiation and depression of synaptic excitation between hippocampal CA3-CA1 cell pairs in vitro. Proc. Natl. Acad. Sci. U.S.A. 93 11225-11230.

Debanne, D., Gähwiler, B. H., and Thompson, S. M. (1998). Long-term synaptic plasticity between pairs of individual CA3 pyramidal cells in rat hippocampal slice cultures. J. Physiol. 507, 237-247.

Debanne, D., Gähwiler, B. H., and Thomspon,S.M.(1999).Heterogeneity of synaptic plasticity at unitary CA3 $\mathrm{CA} 1$ and CA3-CA 3 connections in rat hippocampal slice cultures. J. Neurosci. 19, 10664-10671.

Debanne, D., Guérineau, N.C., Gähwiler, B. H., and Thompson, S. M. (1997) Action-potential propagation gated by an axonal IA-like $\mathrm{K}+$ conductance in hippocampus. Nature 389 286-289.

Dobrunz, L. E., and Stevens, C. F. (1999) Response of hippocampal synapses to natural stimulation patterns. Neuron 22, 157-166.

Dudek, S. M., and Bear, M. F. (1992). Homosynaptic long-term depression in area CAl of hippocampus and effects of N-methyl-D-aspartate receptor blockade. Proc. Natl. Acad. Sci. U.S.A. 89, 4363-4367.

Egger, V., Feldmeyer, D., and Sakmann, B. (1999). Coincidence detection and changes of synaptic efficacy in spiny stellate neurons in rat barrel cortex. Nat. Neurosci. 2, 1098-1105.

Enoki, R., Hu, Y. L., and Fine, A. (2009) Expression of long-term plasticity at individual synapses in hippocampus is graded, bidirectional, and mainly presynaptic: optical quantal analysis. Neuron 62, 242-253.

Feldman, D. E. (2000). Timing-based LTP and LTD at vertical inputs to layer II/ III pyramidal cells in rat barrel cortex. Neuron 27, 45-56.
Feldman, D. E. (2009). Synaptic mechanisms for plasticity in neocortex. Annu. Rev. Neurosci. 32, 33-55.

Franks, K. M., and Sejnowski, T. J. (2002). Complexity of calcium signaling in synaptic spines. Bioessays 24, 1130-1144.

Fregnac, Y., and Shulz, D. E. (1999). Activity-dependent regulation of receptive field properties of cat area 17 by supervised Hebbian learning. $J$. Neurobiol. 41, 69-82.

Froemke, R. C., and Dan, Y. (2002). Spiketiming-dependent synaptic modification induced by natural spike trains. Nature 416, 433-438.

Froemke, R. C., Poo, M.-M., and Dan, Y. (2005). Spike-timing-dependent synaptic plasticity depends on dendritic location. Nature 434, 221-225.

Froemke, R. C., Merzenich, M. M., and Schreiner, C. E. (2007). A synaptic memory trace for cortical receptive field plasticity. Nature 450, 425-429.

Froemke, R. C., Tsay, I. A., Raad, M., Long J. D., and Dan, Y. (2006). Contribution of individual spikes in burst-induced long-term synaptic modification. $J$ Neurophysiol. 95, 1620-1629.

Fusi, S., Drew, P. J., and Abbott, L. F. (2005). Cascade models of synaptically stored memories. Neuron 45, 599-611.

Gerstner, W., Kempter, R., van Hemmen, J. L., and Wagner, H. (1996). A neuronal learning rule for sub-millisecond temporal coding. Nature 383, 76-81.

Gilbert, C. D. (1998). Adult cortical dynamics. Physiol. Rev. 78, 467-485.

Gustafsson, B., Wigstrom, H., Abraham, W. C., and Huang, Y. Y. (1987). Longterm potentiation in the hippocampus using depolarizing current pulses as the conditioning stimulus to single volley synaptic potentials. J. Neurosci. $7,774-780$

Gutig, R., and Sompolinsky, H. (2006) The tempotron: a neuron that learns spike timing-based decisions. Nat. Neurosci. 9, 420-428.

Hansel, C., Artola, A., and Singer, W. (1997). Relation between dendritic $\mathrm{Ca} 2+$ levels and the polarity of synaptic long-term modifications in rat visual cortex neurons. Eur. J. Neurosci. 9, 2309-2322.

Harvey, C. D., and Svoboda, K. (2007). Locally dynamic synaptic learning rules in pyramidal neuron dendrites. Nature 450, 1195-1200.

Hebb, D. O. (1949). The Organization of Behavior. New York, Wiley.

Hoffman, D. A., Magee, J. C., Colbert, C. M., and Johnston, D. (1997). K+ channel regulation of signal propagation in dendrites of hippocampal pyramidal neurons. Nature 387, 869-875.

Hoffman, D. A., Sprengel, R., and Sakmann, B. (2002). Molecular 
dissection of hippocampal theta-burst pairing potentiation. Proc. Natl. Acad. Sci. U.S.A. 99, 7740-7745.

Hopfield, J. J., and Brody, C. D. (2004). Learning rules and network repair in spike-timing-based computation networks. Proc. Natl. Acad. Sci. U.S.A. $101,337-342$.

Izhikevich, E. M., and Edelman, G. M. (2007). Large-scale model of mammalian thalamocortical systems. Proc. Natl. Acad. Sci. U.S.A. 105, 3593-3598.

Johnston, D., Christie, B. R., Frick, A., Gray, R., Hoffman, D. A., Schexnayder, L. K., Watanabe, S., and Yuan, L. L. (2003). Active dendrites, potassium channels and synaptic plasticity. Philos. Trans. R. Soc. Lond. B Biol. Sci. 358, 667-674.

Kampa, B. M., Clements, J., Jonas, P., and Stuart, G. J. (2004). Kinetics of Mg2+ unblock of NMDA receptors: implications for spike-timing dependent plasticity. J. Physiol. 556, 337-345.

Kant, I. (1781). Critique of Pure Reason. New York, Prometheus.

Karmarkar, U. R., and Buonomano, D. V. (2002). A model of spike-timingdependent plasticity: one or two coincidence detectors? J. Neurophysiol. 88, 507-513.

Karmarkar, U. R., Najarian, M. T., and Buonomano,D.V.(2002).Mechanisms and significance of spike-timing dependent plasticity. Biol. Cybern. 87, 373-382.

Katz, L. C., and Shatz, C. J. (1996). Synaptic activity and the construction of cortical circuits. Science 274, 1133-1138.

Kelso, S. R., Ganong, A. H., and Brown, T. H. (1986). Hebbian synapses in hippocampus. Proc. Natl. Acad. Sci. U.S.A. 83, 5326-5330.

Kilgard, M. P., Pandya, P. K., Engineer, N. D., and Moucha, R. (2002). Cortical network reorganization guided by sensory input features. Biol. Cybern. 87, 333-343.

Kirkwood, A., Dudek, S. M., Gold, J. T., Aizenman, C. D., and Bear, M. F. (1993). Common forms of synaptic plasticity in the hippocampus and neocortex in vitro. Science 260, 1518-1521.

Kirkwood, A., Lee, H.-K., and Bear, M. F. (1995). Co-regulation of longterm potentiation and experiencedependent synaptic plasticity in visual cortex by age and experience. Nature 375, 328-331.

Knoblauch, A., and Sommer, F. T. (2003). Synaptic plasticity, conduction delays, and inter-areal phase relations of spike activity in a model of reciprocally connected areas. Neurocomputing 52-54, 301-306.

Koester, H. J., and Sakmann, B. (1998). Calcium dynamics in single spines during coincident pre- and postsynaptic activity depend on relative timing of back-propagating action potentials and subthreshold excitatory postsynaptic potentials. Proc. Natl. Acad. Sci. U.S.A. 95, 9596-9601.

Larkum, M. E., Watanabe, S., Nakamura, T., Lasser-Ross, N., and Ross, W N. (2003). Synaptically activated Ca2+ waves in layer $2 / 3$ and layer 5 rat neocortical pyramidal neurons. J. Physiol. 549, 471-488.

Lee, H.-K., Barbarosie, M., Kameyama, K., Bear, M. F., and Huganir, R. L. (2000). Regulation of distinct AMPA receptor phosphorylation sites during bidirectional synaptic plasticity. Nature 405 , 955-959.

Letzkus, J. J., Kampa, B. M., and Stuart, G. J. (2006). Learning rules for spike timing-dependent plasticity depend on dendritic synapse location. $J$. Neurosci. 26, 10420-10429.

Levy, W. B., and Steward, O. (1979). Synapses as associative memory elements in the hippocampal formation. Brain Res. 175, 233-245.

Levy, W. B., and Steward, O. (1983). Temporal contiguity requirements for long-term associative potentiation/depression in the hippocampus. Neuroscience 8, 791-797.

Lin, M. T., Luján, R., Watanabe, M., Adelman, J. P., and Maylie, J. (2008). SK2 channel plasticity contributes to LTP at Schaffer collateral-CA1 synapses. Nat. Neurosci. 11, 170-177.

Lin, Y. W., Min, M. Y., Chiu, T. H., and Yang, H. W. (2003). Enhancement of associative long-term potentiation by activation of beta-adrenergic receptors at CA1 synapses in rat hippocampal slices. J. Neurosci. 23, 4173-4181.

Lorente de Nó, R. (1938). Synaptic stimulation of motoneurons as a local process. J. Neurophysiol. 1, 195-206.

Magee, J. C., and Johnston, D. (1997). A synaptically controlled, associative signal for Hebbian plasticity in hippocampal neurons. Science 275, 209-213.

Malenka, R. C., and Nicoll, R. A. (1999). Long-term potentiation - a decade of progress? Science 285, 1870-1874.

Markram, H. (2006). The blue brain project. Nat. Rev. Neurosci. 7, 153-160.

Markram, H., Lübke, J., Frotscher, M., and Sakmann, B. (1997). Regulation of synaptic efficacy by coincidence of postsynaptic APs and EPSPs. Science 275, 213-215.

Martin, S. J., Grimwood, P.D., and Morris, R. G. M. (2000). Synaptic plasticity and memory: an evaluation of the hypothesis. Annu. Rev. Neurosci. 23, 649-711.
Meredith, R. M., Floyer-Lea, A. M., and Paulsen, O. (2003). Maturation of long-term potentiation induction rules in rodent hippocampus: role of GABAergic inhibition. J. Neurosci. 23, 11142-11146.

Merzenich,M.M., Jenkins, W.M., Johnston, P., Schreiner, C., Miller, S. L., and Tallal, P. (1996). Temporal processing deficits of language-learning impaired children ameliorated by training. Science 271, 77-81.

Morrison,A., Diesmann, M., and Gerstner, W. (2008). Phenomenological models of synaptic plasticity based on spike timing. Biol. Cybern. 98, 459-478.

Nevian, T., and Sakmann, B. (2006). Spine Ca2+ signaling in spike-timingdependent plasticity. J. Neurosci. 26, 11001-11013.

Nishiyama, M., Hong, K., Mikoshiba, K., Poo, M.-M., and Kato, K. (2000). Calcium stores regulate the polarity and input specificity of synaptic modification. Nature 408, 584-588.

O'Connor, D. H., Wittenberg, G. M., and Wang, S. S. (2005). Graded bidirectional synaptic plasticity is composed of switch-like unitary events. Proc. Natl. Acad. Sci. U.S.A. 102, 9679-9684.

Pagani, M. R., Oishi, K., Gelb, B. D., and Zhong, Y. (2009). The phosphatase SHP2 regulates the spacing effect for long-term memory induction. Cell 139, 186-198.

Paulsen, O., and Sejnowski, T. J. (2000). Natural patterns of activity and longterm synaptic plasticity. Curr. Opin. Neurobiol. 10, 172-179.

Pavlov, I. (1927). Conditioned Reflexes. New York, Dover.

Pawlak, V., and Kerr, J. N. D. (2008). Dopamine receptor activation is required for corticostriatal spiketiming-dependent plasticity. $J$. Neurosci. 28, 2435-2446.

Peterson, C. C., Malenka, R. C., Nicoll, R. A., and Hopfield, J. J. (1998). Allor-none potentiation at CA3-CA1 synapses. Proc. Natl. Acad. Sci. U.S.A. 95, 4732-4737.

Pfister, J.-P., and Gerstner, W. (2006). Triplets of spikes in a model of spike timing-dependent plasticity. J. Neurosci. 26, 9673-9682.

Pike, F. G., Meredith, R. M., Olding, A. W., and Paulsen, O. (1999). Postsynaptic bursting is essential for 'Hebbian' induction of associative long-term potentiation at excitatory synapses in rat hippocampus. J. Physiol. 518, 571-576.

Poncer, J. C., and Malinow, R. (2001). Postsynaptic conversion of silent synapses during LTP affects synaptic gain and transmission dynamics. Nat. Neurosci. 4, 989-996.
Rao, R. P. N., and Sejnowski, T. J. (2001). Spike-timing-dependent Hebbian plasticity as temporal difference learning. Neural. Comput. 13, 2221-2237.

Rasse, T. M., Fouquet, W., Schmid, A., Kittel, R. J., Mertel, S., Sigrist, C. B., Schmidt, M., Guzman, A., Merino, C., Qin, G., Quentin, C., Madeo, F. F., Heckmann, M., and Sigrist, S. J. (2005). Glutamate receptor dynamics organizing synapse formation in vivo. Nat. Neurosci. 8, 898-905.

Reyes, A., and Sakmann, B. (1999). Developmental switch in the short-term modification of unitary EPSPs evoked in layer 2/3 and layer 5 pyramidal neurons of rat neocortex. J. Neurosci. 19, 3827-3835.

Rioult-Pedotti, M.-S., Friedman, D., and Donoghue, J. P. (2000). Learninginduced LTP in neocortex. Science 290, 533-536.

Rubin, J., Lee, D. D., and Sompolinsky, H. (2001). Equilibrium properties of temporally asymmetric Hebbian plasticity. Phys. Rev. Lett. 86, 364-367.

Rubin, J. E., Gerkin, R. C., Bi, G.-Q., and Chow, C. C. (2005). Calcium time course as a signal for spike-timingdependent plasticity. J. Neurophysiol. 93, 2600-2613.

Schafe, G. E., Nader, K., Blair, H. T., and LeDoux, J.E. (2001). Memory consolidation of Pavlovian fear conditioning: a cellular and molecular perspective. Trends Neurosci. 24, 540-546.

Scharfman, H.E., and Sarvey, J.M. (1985). Postsynaptic firing during repetitive stimulation is required for long-term potentiation in hippocampus. Brain Res. 331, 267-274.

Sejnowski, T. J. (1999). The book of Hebb. Neuron 24, 773-776.

Senn, W., Markram H., and Tsodyks, M. (1999). An algorithm for modifying neurotransmitter release probability based on pre- and postsynaptic spike timing. Neural. Comput. 13, 35-67.

Seol, G. H., Ziburkus, J., Huang, S., Song, L., Kim, I. T., Takamiya, K., Huganir, R. L., Lee, H. K., and Kirkwood, A. (2007). Neuromodulators control the polarity of spike-timing-dependent-synaptic plasticity. Neuron 55 , 919-929.

Shen, W., Flajolet, M., Greengard, P., and Surmeier, D. J. (2008). Dichotomous dopaminergic control of striatal synaptic plasticity. Science 321, 848-851.

Shouval, H. Z., Bear, M. F., and Cooper, L. N. (2002). A unified model of NMDA receptor-dependent bidirectional synaptic plasticity. Proc. Natl. Acad. Sci. U.S.A. 99, 10831-10836.

Sjöström, P. J., and Häusser M. (2006). A cooperative switch determines the sign of synaptic plasticity in distal dendrites of neocortical pyramidal neurons. Neuron 51, 227-238. 
Sjöström, P. J., Turrigiano, G. G., and Nelson S. B. (2001). Rate, timing, and cooperativity jointly determine cortical synaptic plasticity. Neuron 32, 1149-1164.

Sjöström, P. J., Turrigiano, G. G., and Nelson, S. B. (2003). Neocortical LTD via coincident activation of presynaptic NMDA and cannabinoid receptors. Neuron 39, 641-654.

Sjöström, P. J., Turrigiano, G. G., and Nelson, S. B. (2004). Endocannabinoiddependent neocortical layer-5 LTD in the absence of postsynaptic spiking. J. Neurophysiol. 92, 3338-3343.

Song, S., Miller, K. D., and Abbott, L. F. (2000). Competitive Hebbian learning through spike-timing-dependent synaptic plasticity. Nat. Neurosci. 3, 919-926.

Sourdet, V., and Debanne, D. (1999). The role of dendritic filtering in associative long-term synaptic plasticity. Learn. Mem. 6, 422-447.

Stanton, P. K., and Sejnowski, T. J. (1989). Associative long-term depression in the hippocampus induced by Hebbian covariance. Nature 339, 215-218.

Stent, G. S. (1973). A physiological mechanism for Hebb's postulate of learning. Proc. Natl. Acad. Sci. U.S.A. 70, 997-1001.

Sutton, R. S., and Barto, A. G. (1998). Reinforcement Learning: An Introduction. Boston, The MIT Press.

Tallal, P., Miller, S. L., Bedi, G., Byma, G., Wang, X., Nagarajan, S. S., Schreiner, C., Jenkins, W. M., and Merzenich, M. M. (1996). Language comprehension in language-learning impaired children improved with acoustically modified speech. Science 271, 81-84.

Tanaka, E., Higashi, H., and Nishi, S. (1991). Membrane properties of guinea pig cingulate cortical neurons in vitro. J. Neurophysiol. 65, 808-821.

Tanaka, J.-I., Horiike, Y., Matsuzaki, M., Miyazaki, T., Ellis-Davies, G. C. R., and Kasai, H. (2008). Protein synthesis and neurotrophin-dependent structural plasticity of single dendritic spines. Science 319, 1683-1687.

Tsukada, M., Aihara, T., Kobayashi, Y., and Shimazaki, H. (2005). Spatial analysis of spike-timing-dependent LTP and LTD in the CA1 area of hippocampal slices using optical imaging. Hippocampus 15, 104-109.

Turrigiano, G. G., and Nelson, S. B. (2000). Hebb and homeostasis in neuronal plasticity. Curr. Opin. Neurobiol. 10, 358-364.

Tzounopoulos, T., Kim, Y., Oertel, D., and Trussell, L. O. (2004). Cell-specific, spike timing-dependent plasticities in the dorsal cochlear nucleus. Nat. Neurosci. 7, 719-725.

Tzounopoulos, T., Rubio, M. E., T., Keen, J. E., and Trussell, L. O. (2007). Coactivation of pre- and postsynaptic signaling mechanisms determines cell-specific spike-timing-dependent plasticity. Neuron 54, 291-301.

Urakubo, H., Honda, M., Froemke, R. C., and Kuroda, S. (2008). Requirement of an allosteric kinetics of NMDA receptors for spike timing-dependent plasticity. J. Neurosci. 28, 3310-3323.

van Rossum, M. C. W., Bi, G.-Q., and Turrigiano, G. G. (2000). Stable Hebbian learning from spike timingdependent plasticity. J. Neurosci. 20, 8812-8821.

Varela, J. A., Sen, K., Gibson, J., Fost, J., Abbott, L. F., and Nelson, S. B. (1997). A quantitative description of shortterm plasticity at excitatory synapses in layer $2 / 3$ of rat primary visual cortex. J. Neurosci. 17, 7926-7940.

Wang, H.-X., Gerkin, R. C., Nauen, D. W., and Bi, G.-Q. (2005). Coactivation and timing-dependent integration of synaptic potentiation and depression. Nat. Neurosci. 8, 187-193.

Wang, S. S., Denk, W., and Häusser, M. (2000). Coincidence detection in single dendritic spines mediated by calcium release. Nat. Neurosci. 3, 1266-1273.

Watanabe, S., Hoffman, D. A., Migliore, M., and Johnston, D. (2002). Dendritic $\mathrm{K}+$ channels contribute to spiketiming dependent long-term potentiation in hippocampal pyramidal neurons. Proc. Natl. Acad. Sci. U.S.A. 99, 8366-8371.

Wittenberg, G. M., and Wang, S. S. (2006). Malleability of spike-timing-dependent plasticity at the CA3-CA1 synapse. J. Neurosci. 26, 6610-6617.

Yang, S. N., Tang, Y. G., and Zucker, R. S. (1999). Selective induction of LTP and LTD by postsynaptic [Ca2+]i elevation. J. Neurophysiol. 81, 781-787.

Zador, A., Koch, C., and Brown, T. H. (1990). Biophysical model of a Hebbian synapse. Proc. Natl. Acad. Sci. U.S.A. 87, 6718-6722.

Zhang, J. C., Lau, P. M., and Bi, G.-Q. (2009). Gain in sensitivity and loss in temporal contrast of STDP by dopaminergic modulation at hippocampal synapses. Proc. Natl. Acad. Sci. U.S.A. 106, 13028-13033.

Zhang, L. I., Tao, H. W., Holt, C. E., Harris, W. A., and Poo, M. (1998). A critical window for cooperation and competition among developing retinotectal synapses. Nature 395, 37-44.

Zhou, Q., Tao, H. W., and Poo, M. M. (2003). Reversal and stabilization of synaptic modifications in a developing nervous system. Science 300 , 1953-1957.

Zilberter, M., Holmgren, C., Shemer, I., Silberberg, G., Grillner, S., Harkany, T., and Zilberter, Y. (2009). Input specificity and dependence of spike timing-dependent plasticity on preceding postsynaptic activity at unitary connections between neocortical layer 2/3 pyramidal cells. Cereb. Cortex 19, 2308-2320.

Zucker, R. S. (1999). Calcium- and activity-dependent synaptic plasticity. Curr. Opin. Neurobiol. 9, 305-313.

Zucker, R. S., and Regehr, W. G. (2002). Short-term synaptic plasticity. Annu. Rev. Physiol. 64, 355-405.

Conflict-of-Interest Statement: The authors declare that the research was conducted in the absence of any commercial or financial relationships that could be construed as a potential conflict of interest.

Received: 01 February 2010; paper pending published: 30 April 2010; accepted: 27 May 2010; published online: 17 June 2010.

Citation: Froemke RC, Debanne D and Bi G-Q (2010) Temporal modulation of spike-timing-dependent plasticity. Front. Syn. Neurosci. 2:19. doi: 10.3389/ fnsyn.2010.00019

Copyright (c) 2010 Froemke, Debanne and $B i$. This is an open-access article subject to an exclusive license agreement between the authors and the Frontiers Research Foundation, which permits unrestricted use, distribution, and reproduction in any medium, provided the original authors and source are credited. 